\title{
Emerging drugs of abuse: current perspectives on synthetic cannabinoids
}

This article was published in the following Dove Press journal:

Substance Abuse and Rehabilitation

20 October 2015

Number of times this article has been viewed

\author{
Danièle Debruyne ${ }^{1,2}$ \\ Reynald Le Boisselier' \\ 'Centre for Evaluation and \\ Information on Pharmacodependence - \\ Addictovigilance (CEIP-A), \\ ${ }^{2}$ Toxicology and Pharmacology \\ Laboratory, Department of \\ Pharmacology, University Hospital \\ Centre Côte de Nacre, Caen, France
}

\begin{abstract}
New psychoactive drugs that have appeared over the last decade are typically dominated by cathinones and synthetic cannabinoids (SCs). SCs have been emerging as recreational drugs because they mimic the euphoria effect of cannabis while still being legal. Sprayed on natural herb mixtures, SCs have been primarily sold as "herbal smoking blends" or "herbal incense" under brand names like "Spice" or "K2". Currently, SCs pure compounds are available from websites for the combination with herbal materials or for the use in e-cigarettes. For the past 5 years, an ever increasing number of compounds, representative of different chemical classes, have been promoted and now represent a large assortment of new popular drugs of abuse, which are difficult to properly identify. Their legal status varies by country with many government institutions currently pushing for their control. The in vitro binding to $\mathrm{CB} 1 / \mathrm{CB} 2$ receptors is usually well-known and considerable differences have been found in the CB1 versus CB2 selectivity and potency within the different SCs, with several structure-activity relations being evident. Desired effects by CB1 agonist users are relaxation/recreative, however, cardiovascular, gastrointestinal, or psychiatric/neurological side effects are commonly reported. At present there is no specific antidote existing if an overdose of designer drugs was to occur, and no curative treatment has been approved by health authorities. Management of acute toxic effects is mainly symptomatic and extrapolated from experience with cannabis.
\end{abstract}

Keywords: synthetic cannabinoids, chemistry, analysis, pharmacology, toxicology, dependence, medical care

\section{Introduction}

Synthetic cannabinoids (SCs) were originally developed in the 1970's for the research on ligands and the exploration of their pharmacological endocannabinoid pathways. ${ }^{1}$ Just like the primary psychoactive component of cannabis, $\Delta^{9}$-tetrahydrocannabinol $\left(\Delta^{9}\right.$-THC), and in the same manner as the main endogenous ligands, anandamide, and 2-arachydonylglycerol, SCs bind to the two subtypes of cannabinoid receptors, $\mathrm{CB} 1$ and $\mathrm{CB} 2$, with a varying degree of affinity. SCs were first reported for their use in recreational settings in December 2008 by a German firm who identified them in the smoking blends sold as herbs sprayed or mixed with one or more synthetic compounds, and were referred to as "Spice", "Yucatan", "Chill”, "K2", or "Black Mamba".

Over the following years SCs gained in popularity, especially among young people. Proposed as medicinal and legal products, newly synthetized cannabimimetics continue to emerge on the "legal highs" market as an alternative to phytocannabinoids. Among SCs, CB1 agonists mimic the effects of cannabis where consumers may feel happy and relaxed. However, unwanted serious adverse effects, such as neuropsychiatric disturbances or
Correspondence: Danièle Debruyne Department of Pharmacology, University Hospital Centre Côte de Nacre, I 4033

Caen cedex 9 , France

Tel +3323I 064641

$\mathrm{Fax}+33231064673$

Email debruyne-d@chu-caen.fr 
somatic effects of variable intensity, may occur with the use of recreational SCs. ${ }^{3}$ Moreover, as result of the various forms of synaptic plasticity mediated by endocannabinoids, which allows the cannabinoid receptor to recognize multiple classes of compounds, ${ }^{4}$ a large variety of distinct chemical substances are now sold over the Internet. This reinforces the public health problem linked to these new SCs that have, for the greater part, never been tested in human controlled settings. Nevertheless, as ever-increasing data on the identification or analysis methods, pharmacokinetics or pharmacodynamics, animal or human pharmacology or toxicity, and addictive potential are becoming available, this review is aimed to present updated information on SCs, useful for poisons centers, clinical toxicologists and emergency physicians.

\section{Availability and usage demographics}

SCs were first developed with the aim of exploring endogenous cannabinergic pathways and finding new therapeutics. ${ }^{5}$ In the mid-1970s, Pfizer created CP 55,940, and in the 1980's and 1990's, other chemicals such as HU-210 and WIN 55,212 were investigated as potent pharmacotherapies. ${ }^{6}$ The most important series of SCs appeared from the study by Huffman and Dong in 1994. ${ }^{7}$ From this time on new classes of SCs have been developed, with numerous representatives inside every drug class.

The history on the use of SCs as drugs of abuse began approximately around 2004 when herbal mixtures, mainly known as Spice, were marketed on the Internet as a substitute to cannabis, in colorful attractive packages. Initially, these blends seemed to be made from plants traditionally used by shamans and/or other well known "phyto-chemistry" adepts. Four years later, in December 2008, the first reported case of SCs misuse appeared with the discovery of five compounds (JWH-018, CP 47,497 and its C6, C7, C8 analogs) in herbal blends produced by a German company. ${ }^{2}$ Neither the seller nor the consumer of herbal mixtures, such as Spice, K2, or Black Mumba, can predict their content. For example, the overall range of concentrations in nine different brands investigated by Lindigkeit et al was between 3-11 mg/g of CP 47,497 C8, and 6-23 mg/g of JWH-073. One sample contained a small amount of JWH-018, ie, $2.3 \mathrm{mg} / \mathrm{g}$, while two other samples were found to be free of SCs. ${ }^{8}$ Similar findings were published in another study by Uchiyama et al based on 46 Spice products. ${ }^{9}$ Concomitantly with the emergence of herbal blends, SCs quickly become available in large amounts of "pure" powder, sold especially on websites from the People's Republic of China, with the aim of adding to tobacco for smoking.

Nowadays, new SCs constantly appear on the market, ${ }^{10}$ along with new types of consumption, which could be called a third-generation of use, such as cartridges filled with SCs solution designed for using with e-cigarettes. These are called "buddha-blue", "C-Liquid", "Herbal e-Liquid", or others and are discussed on drug-user forums.

From published data available from poisoning, toxicological, or epidemiological centers the use of SCs is common in the USA, varying from $1.4 \%$ based on Wohlfarth et al's study on USA military urine specimens $(n=20,017)$ collected from July 2011 to June $2012,{ }^{11}$ up to approximately $10 \%$ found in Palamar and Acosta's study taken on high school seniors $(n=11863) .{ }^{12}$ Similar findings were reported from studies on the USA nightlife scene $(n=1740)^{13}$ and on college students in Florida $(n=852) .{ }^{14}$ However, it has been recently shown that while lifetime prevalence has increased, the past 6 month prevalence has decreased substantially over time. ${ }^{15}$ In Europe, a retrospective study based on serum samples collected in Germany in 2010, estimated a prevalence at approximately $2.8 \%(n=12 / 422),{ }^{16}$ and $4 \%(n=164 / 4080)$ in a youth population (18-34 year olds) from the French Health Barometer. ${ }^{17}$ Considering the new attraction for SCs, studies or reports on driving impairment have started to be considered in USA ${ }^{18,19}$ and in Europe..$^{20,21}$

It is also important to note that SCs can be used as a therapeutic. Even if many fundamental research is conducted, only a few SCs are available for medical use, belonging to dibenzopyrane derivatives such as nabilone or dronabinol. They are mostly used for their antiemetic properties, especially in chemotherapy-induced nausea and vomiting, or for their orexigenic properties in anorexia. In addition, analgesic properties of cannabinoids are advanced in some Cannabis sativa plant extracts for adjunctive treatment of neuropathic pain in patients with multiple sclerosis. To date, several investigations are currently underway to find new therapeutic applications of SCs, for example, neuroprotective effects in neurodegenerative diseases such as Parkinson's disease, ${ }^{22}$ or inflammatory cell modulation. ${ }^{23}$

\section{Chemical structure and designation}

SCs family is extremely large, including numerous substances belonging to various chemical groups and subgroups. New compounds which can belong to unknown chemical classes emerge constantly, making the inventory of existing products never ending. We have brought together approximately 120 SCs, which are the most recent and popular compounds, but we do not claim this list to be exhaustive (Tables 1 and 2).

We specify trade names, radicals, formula, and CAS numbers for an easy and quicker tracking. Only a few SCs are structurally related to $\Delta^{9}-\mathrm{THC}$, the others belonging to 
Table I Chemical structure of cannabinoid 3-indole derivatives

\begin{tabular}{|c|c|c|c|c|c|c|c|}
\hline Name & $\mathbf{R}$ & $\mathbf{R}^{\prime}$ & $\mathbf{R} \mathbf{I}$ & $\mathbf{R 2}$ & $\mathbf{R 3}$ & Formula & CAS number \\
\hline \multicolumn{8}{|l|}{ Benzoyl derivatives } \\
\hline AM-679 & & 2-iodo & Pentyl & $\mathrm{H}$ & $\ldots$ & $\mathrm{C} 2 \mathrm{OH} 2 \mathrm{OINO}$ & $335160-91-3$ \\
\hline AM-694 & & 2-iodo & 5-fluoropentyl & $\mathrm{H}$ & $\ldots$ & C2OHI9FINO & $335161-03-0$ \\
\hline $\mathrm{RCS}-4=\mathrm{SR} 19=\mathrm{E}$ & & 4-methoxy & Pentyl & $\mathrm{H}$ & $\ldots$ & $\mathrm{C} 21 \mathrm{H} 23 \mathrm{NO} 2$ & | 345966-78-0 \\
\hline \multicolumn{8}{|l|}{$4=$ BTM $4=$ OBT 199} \\
\hline WIN-48,098 = pravadoline & & 4-methoxy & nos & Methyl & $\ldots$ & $\mathrm{C} 23 \mathrm{H} 26 \mathrm{~N} 2 \mathrm{O} 3$ & $92623-83-1$ \\
\hline AM-2233 & & 2-iodo & $\tau$ & $\mathrm{H}$ & $\ldots$ & $\mathrm{C} 22 \mathrm{H} 23 \mathrm{IN} 2 \mathrm{O}$ & $444912-75-8$ \\
\hline RCS-8 & & 2-methoxy & no & $\mathrm{H}$ & $\ldots$ & $\mathrm{C} 25 \mathrm{H} 29 \mathrm{NO} 2$ & | 345970-42-4 \\
\hline \multicolumn{8}{|l|}{ Naphthoyl derivatives } \\
\hline JWH-007 & & $\ldots$ & Pentyl & Methyl & $\ldots$ & $\mathrm{C} 25 \mathrm{H} 25 \mathrm{NO}$ & $|5547|-10-6$ \\
\hline JWH-0I5 & & $\ldots$ & Propyl & Methyl & $\ldots$ & $\mathrm{C} 23 \mathrm{H} 2 \mathrm{INO}$ & |5547|-08-2 \\
\hline JWH-0I8 & & $\ldots$ & Pentyl & $\mathrm{H}$ & $\ldots$ & $\mathrm{C} 24 \mathrm{H} 23 \mathrm{NO}$ & $2094 \mid 4-07-3$ \\
\hline JWH-0I9 & & $\ldots$ & Hexyl & $\mathrm{H}$ & $\ldots$ & $\mathrm{C} 25 \mathrm{H} 25 \mathrm{NO}$ & $2094 \mid 4-08-4$ \\
\hline JWH-022 & & $\ldots$ & Pentenyl & $\mathrm{H}$ & $\ldots$ & $\mathrm{C} 24 \mathrm{H} 2 \mathrm{INO}$ & $2094 \mid 4-16-4$ \\
\hline JWH-07I & & $\ldots$ & Ethyl & $\mathrm{H}$ & $\ldots$ & $\mathrm{C} 2 \mathrm{IHI}$ NNO & $2094 \mid 4-05-1$ \\
\hline JWH-073 & & $\ldots$ & Butyl & $\mathrm{H}$ & $\ldots$ & $\mathrm{C} 23 \mathrm{H} 2 \mathrm{INO}$ & $208987-48-8$ \\
\hline JWH-08I & & 4-methoxy & Pentyl & $\mathrm{H}$ & $\ldots$ & $\mathrm{C} 25 \mathrm{H} 25 \mathrm{NO} 2$ & $210179-46-7$ \\
\hline JWH-098 & & 4-methoxy & Pentyl & Methyl & $\ldots$ & $\mathrm{C} 26 \mathrm{H} 27 \mathrm{NO} 2$ & $316189-79-9$ \\
\hline JWH-II6 & & $\ldots$ & Pentyl & Ethyl & $\ldots$ & $\mathrm{C} 26 \mathrm{H} 27 \mathrm{NO}$ & 619294-64-3 \\
\hline JWH-I22 & & 4-methyl & Pentyl & $\mathrm{H}$ & $\ldots$ & $\mathrm{C} 25 \mathrm{H} 25 \mathrm{NO}$ & $619294-47-2$ \\
\hline JWH-I49 & & 4-methyl & Pentyl & Methyl & $\ldots$ & $\mathrm{C} 26 \mathrm{H} 27 \mathrm{NO}$ & $54846 \mid-82-1$ \\
\hline JWH-I82 & & 4-propyl & Pentyl & $\mathrm{H}$ & $\ldots$ & $\mathrm{C} 27 \mathrm{H} 29 \mathrm{NO}$ & $824960-02-3$ \\
\hline JWH-I93 & & 4-methyl & $\sim \infty$ & $\mathrm{H}$ & $\ldots$ & $\mathrm{C} 26 \mathrm{H} 26 \mathrm{~N} 2 \mathrm{O} 2$ & | 33438-58-| \\
\hline JWH-198 & & 4-methoxy & $\sim \infty$ & $\mathrm{H}$ & $\ldots$ & $\mathrm{C} 26 \mathrm{H} 26 \mathrm{~N} 2 \mathrm{O} 3$ & $166599-76-4$ \\
\hline JWH-200 = WIN 55,225 & & $\ldots$ & $\cdots$ & $\mathrm{H}$ & $\ldots$ & $\mathrm{C} 25 \mathrm{H} 24 \mathrm{~N} 2 \mathrm{O} 2$ & $103610-04-4$ \\
\hline JWH-2I0 & & 4-ethyl & Pentyl & $\mathrm{H}$ & $\ldots$ & $\mathrm{C} 26 \mathrm{H} 27 \mathrm{NO}$ & $824959-81-1$ \\
\hline JWH-387 & & 4-bromo & Pentyl & $\mathrm{H}$ & $\ldots$ & $\mathrm{C} 24 \mathrm{H} 22 \mathrm{BrNO}$ & $1366067-59-5$ \\
\hline JWH-398 & & 4-chloro & Pentyl & $\mathrm{H}$ & $\ldots$ & $\mathrm{C} 24 \mathrm{H} 22 \mathrm{CINO}$ & $\mid 292765-18-4$ \\
\hline $\mathrm{JWH}-4 \mathrm{I2}$ & & 4-fluoro & Pentyl & $\mathrm{H}$ & $\ldots$ & $\mathrm{C} 24 \mathrm{H} 22 \mathrm{FNO}$ & | 364933-59-4 \\
\hline JWH-424 & & 8-bromo & Pentyl & $\mathrm{H}$ & $\ldots$ & $\mathrm{C} 24 \mathrm{H} 22 \mathrm{BrNO}$ & |366068-04-3 \\
\hline AM-I220 & & $\ldots$ & $\tau^{*}$ & $\mathrm{H}$ & $\ldots$ & $\mathrm{C} 26 \mathrm{H} 26 \mathrm{~N} 2 \mathrm{O}$ & | 37642-54-7 \\
\hline AM-I22I & & $\ldots$ & 光 & Methyl & $\mathrm{NO} 2$ & $\mathrm{C} 27 \mathrm{H} 27 \mathrm{~N} 3 \mathrm{O} 3$ & $335160-53-7$ \\
\hline AM- 1235 & & $\ldots$ & 5-fluoropentyl & $\mathrm{H}$ & $\mathrm{NO} 2$ & $\mathrm{C} 24 \mathrm{H} 2 \mathrm{IFN} 2 \mathrm{O} 3$ & $33516 \mid-27-8$ \\
\hline AM-220I & & $\ldots$ & 5-fluoropentyl & $\mathrm{H}$ & $\ldots$ & $\mathrm{C} 24 \mathrm{H} 22 \mathrm{FNO}$ & $33516 \mid-24-5$ \\
\hline AM-2232 & & $\ldots$ & 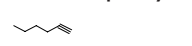 & $\mathrm{H}$ & $\ldots$ & $\mathrm{C} 24 \mathrm{H} 20 \mathrm{~N} 2 \mathrm{O}$ & $335161-19-8$ \\
\hline MAM-220I & & 4-methyl & 5-fluoropentyl & $\mathrm{H}$ & $\ldots$ & $\mathrm{C} 25 \mathrm{H} 24 \mathrm{FNO}$ & | $35463 \mid-24-5$ \\
\hline EAM-220I & & 4-ethyl & 5-fluoropentyl & $\mathrm{H}$ & $\ldots$ & $\mathrm{C} 26 \mathrm{H} 26 \mathrm{FNO}$ & | 364933-60-7 \\
\hline \multicolumn{8}{|l|}{ Phenylacetyl derivatives } \\
\hline JWH-I67 & & $\ldots$ & Pentyl & $\mathrm{H}$ & $\ldots$ & $\mathrm{C} 2 \mathrm{IH} 23 \mathrm{NO}$ & $864445-37-4$ \\
\hline JWH-20I & & 4-methoxy & Pentyl & $\mathrm{H}$ & $\ldots$ & $\mathrm{C} 22 \mathrm{H} 25 \mathrm{NO} 2$ & $864445-47-6$ \\
\hline JWH-203 & & 2-chloro & Pentyl & $\mathrm{H}$ & $\ldots$ & $\mathrm{C} 2 \mathrm{IH} 22 \mathrm{CINO}$ & $864445-54-5$ \\
\hline JWH-204 & & 2-chloro & Pentyl & Methyl & $\ldots$ & $\mathrm{C} 22 \mathrm{H} 24 \mathrm{CINO}$ & $864445-55-6$ \\
\hline JWH-206 & & 4-chloro & Pentyl & $\mathrm{H}$ & $\ldots$ & $\mathrm{C} 2 \mathrm{IH} 22 \mathrm{CINO}$ & $864445-58-9$ \\
\hline JWH-207 & & 4-chloro & Pentyl & Methyl & $\ldots$ & $\mathrm{C} 22 \mathrm{H} 24 \mathrm{CINO}$ & $864445-59-0$ \\
\hline JWH-208 & & 4-methyl & Pentyl & $\mathrm{H}$ & $\ldots$ & $\mathrm{C} 22 \mathrm{H} 25 \mathrm{NO}$ & $864445-4 \mid-0$ \\
\hline JWH-209 & & 4-methyl & Pentyl & Methyl & $\ldots$ & $\mathrm{C} 23 \mathrm{H} 27 \mathrm{NO}$ & $864445-42-1$ \\
\hline JWH-249 & & 2-bromo & Pentyl & $\mathrm{H}$ & $\ldots$ & $\mathrm{C} 2 \mathrm{IH} 22 \mathrm{BrNO}$ & $864445-60-3$ \\
\hline JWH-250 & & 2-methoxy & Pentyl & $\mathrm{H}$ & $\ldots$ & $\mathrm{C} 22 \mathrm{H} 25 \mathrm{NO} 2$ & $864445-43-2$ \\
\hline JWH-25I & & 2-methyl & Pentyl & $\mathrm{H}$ & $\ldots$ & $\mathrm{C} 22 \mathrm{H} 25 \mathrm{NO} 2$ & $864445-39-6$ \\
\hline JWH-253 & & 3-methoxy & Pentyl & Methyl & $\ldots$ & $\mathrm{C} 23 \mathrm{H} 27 \mathrm{NO} 2$ & $864445-46-5$ \\
\hline $\mathrm{JWH}-302$ & & 3-methoxy & Pentyl & $\mathrm{H}$ & $\ldots$ & $\mathrm{C} 22 \mathrm{H} 25 \mathrm{NO} 2$ & $864445-45-4$ \\
\hline JWH-3II & & 2-fluoro & Pentyl & $\mathrm{H}$ & $\ldots$ & $\mathrm{C} 2 \mathrm{IH} 22 \mathrm{FNO}$ & $864445-49-8$ \\
\hline JWH-316 & & 4-fluoro & Pentyl & Methyl & $\ldots$ & $\mathrm{C} 22 \mathrm{H} 24 \mathrm{FNO}$ & $864445-53-4$ \\
\hline
\end{tabular}


Table I (Continued)

\begin{tabular}{|c|c|c|c|c|c|c|c|}
\hline Name & $\mathbf{R}$ & $\mathbf{R}^{\prime}$ & $\mathbf{R} \mathbf{I}$ & $\mathbf{R 2}$ & $\mathbf{R 3}$ & Formula & CAS number \\
\hline \multicolumn{8}{|l|}{ Alkoyl derivatives } \\
\hline A-790,260 & & $\Varangle$ & $\sim v_{0}$ & $\mathrm{H}$ & $\ldots$ & $\mathrm{C} 22 \mathrm{H} 30 \mathrm{~N} 2 \mathrm{O} 2$ & $895155-26-7$ \\
\hline A-834,735 & & $-x$ & 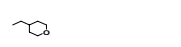 & $\mathrm{H}$ & $\ldots$ & $\mathrm{C} 22 \mathrm{H} 29 \mathrm{NO} 2$ & $895155-57-4$ \\
\hline AB-005 & & $-\Varangle$ & $\gamma^{i}$ & $\mathrm{H}$ & $\ldots$ & $\mathrm{C} 23 \mathrm{H} 32 \mathrm{~N} 2 \mathrm{O}$ & $895 \mid 555-25-6$ \\
\hline UR-I44 & & $-\Varangle$ & Pentyl & $\mathrm{H}$ & $\ldots$ & $\mathrm{C} 2 \mathrm{IH} 29 \mathrm{NO}$ & I I 99943-44-6 \\
\hline XLR-II = 5F-UR-I44 & & $\Varangle$ & 5-fluoropentyl & $\mathrm{H}$ & $\ldots$ & $\mathrm{C} 2 \mathrm{IH} 28 \mathrm{FNO}$ & 1364933-54-9 \\
\hline AB-00I & & $\pi$ & Pentyl & $\mathrm{H}$ & $\ldots$ & $\mathrm{C} 24 \mathrm{H} 3 \mathrm{INO}$ & $1345973-49-0$ \\
\hline AM-I 248 & & $\Theta$ & $\tau^{2}$ & $\mathrm{H}$ & $\ldots$ & $\mathrm{C} 26 \mathrm{H} 34 \mathrm{~N} 2 \mathrm{O}$ & $335160-66-2$ \\
\hline \multicolumn{8}{|l|}{ Piperazoyl derivatives } \\
\hline Mepirapim & ${ }_{Y} \sqrt[N]{N}-R^{\prime}$ & Methyl & Pentyl & $\mathrm{H}$ & $\ldots$ & $\mathrm{Cl} 9 \mathrm{H} 27 \mathrm{~N} 3 \mathrm{O}$ & Not attributed \\
\hline \multicolumn{8}{|l|}{ Carboxylate derivatives } \\
\hline BB-22 = QUCHIC & & 20 & $\infty$ & $\mathrm{H}$ & $\ldots$ & $\mathrm{C} 25 \mathrm{H} 24 \mathrm{~N} 2 \mathrm{O} 2$ & | 400742-42-8 \\
\hline PB-22 = QUPIC & & $\alpha_{\infty}$ & Pentyl & $\mathrm{H}$ & $\ldots$ & $\mathrm{C} 23 \mathrm{H} 22 \mathrm{~N} 2 \mathrm{O} 2$ & |400742-| 7-7 \\
\hline $5 F-P B-22$ & & des & 5-fluoropentyl & $\mathrm{H}$ & $\ldots$ & $\mathrm{C} 23 \mathrm{H} 2 \mathrm{IFN} 2 \mathrm{O} 2$ & $|400742-4|-7$ \\
\hline FUB-PB-22 & & bo & $\neg-F$ & $\mathrm{H}$ & $\ldots$ & $\mathrm{C} 25 \mathrm{HI} 7 \mathrm{FN} 2 \mathrm{O} 2$ & Not attributed \\
\hline FDU-PB-22 & & $\infty$ & 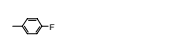 & $\mathrm{H}$ & $\ldots$ & $\mathrm{C} 26 \mathrm{HI} 8 \mathrm{FNO} 2$ & Not attributed \\
\hline NM-220I = CBL-220I & & $\infty$ & 5-fluoropentyl & $\mathrm{H}$ & $\ldots$ & $\mathrm{C} 24 \mathrm{H} 22 \mathrm{FNO} 2$ & Not attributed \\
\hline \multicolumn{8}{|l|}{ Carboxamide derivatives } \\
\hline ADBICA & & $x_{1} i_{\mathrm{Nu}_{2}}$ & Pentyl & $\mathrm{H}$ & $\ldots$ & $\mathrm{C} 20 \mathrm{H} 29 \mathrm{~N} 3 \mathrm{O} 2$ & |445583-48-| \\
\hline CUMYL-BICA & & $x_{0}$ & Butyl & $\mathrm{H}$ & $\ldots$ & $\mathrm{C} 22 \mathrm{H} 26 \mathrm{~N} 2 \mathrm{O}$ & Not attributed \\
\hline CUMYL-PICA & & $x_{0}$ & Pentyl & $\mathrm{H}$ & $\ldots$ & $\mathrm{C} 23 \mathrm{H} 28 \mathrm{~N} 2 \mathrm{O}$ & Not attributed \\
\hline CUMYL-5FPICA & & $x_{0}$ & 5-fluoropentyl & $\mathrm{H}$ & $\ldots$ & $\mathrm{C} 23 \mathrm{H} 27 \mathrm{FN} 2 \mathrm{O}$ & Not attributed \\
\hline MDMB-CHMICA = MMB-CHMINACA & & $x^{2}$ & $\uparrow$ & $\mathrm{H}$ & $\ldots$ & $\mathrm{C} 23 \mathrm{H} 32 \mathrm{~N} 2 \mathrm{O} 3$ & $832231-92-2$ \\
\hline $\mathrm{NNEI}=\mathrm{MN} 24$ & & do & Pentyl & $\mathrm{H}$ & $\ldots$ & $\mathrm{C} 24 \mathrm{H} 24 \mathrm{~N} 2 \mathrm{O}$ & |338925-||-3 \\
\hline$M N-25=U R-12$ & & 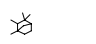 & $\sim 2$ & $\mathrm{H}$ & Methoxy & $\mathrm{C} 26 \mathrm{H} 37 \mathrm{~N} 3 \mathrm{O} 3$ & $501926-82-5$ \\
\hline $5 F-N N E I=5 F-M N 24$ & & b & 5-fluoropentyl & $\mathrm{H}$ & $\ldots$ & $\mathrm{C} 24 \mathrm{H} 23 \mathrm{FN} 2 \mathrm{O}$ & |445580-60-8 \\
\hline SDB-00I = APICA = 2-NEI & & $\otimes$ & Pentyl & $\mathrm{H}$ & $\ldots$ & $\mathrm{C} 24 \mathrm{H} 32 \mathrm{~N} 2 \mathrm{O}$ & $1345973-50-3$ \\
\hline STS- 135 & & $\otimes$ & 5-fluoropentyl & $\mathrm{H}$ & $\ldots$ & $\mathrm{C} 24 \mathrm{H} 3 \mathrm{IN} 2 \mathrm{O}$ & $|35463|-26-7$ \\
\hline SDB-006 & & 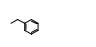 & Pentyl & $\mathrm{H}$ & $\ldots$ & $\mathrm{C} 2 \mathrm{IH} 24 \mathrm{~N} 2 \mathrm{O}$ & $695213-59-3$ \\
\hline 5F-SDB-006 & & 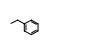 & 5-fluoropentyl & $\mathrm{H}$ & $\ldots$ & $\mathrm{C} 2 \mathrm{IH} 23 \mathrm{FN} 2 \mathrm{O}$ & Not attributed \\
\hline SRF-30 = PX-I = 5F-APP-PICA & & $x_{0}$ & 5-fluoropentyl & $\mathrm{H}$ & $\ldots$ & $\mathrm{C} 23 \mathrm{H} 26 \mathrm{FN} 3 \mathrm{O} 2$ & Not attributed \\
\hline \multicolumn{8}{|l|}{ Thiazolyl derivatives } \\
\hline PTI- I & & $r^{-N} \sqsubset$ & Pentyl & $\mathrm{H}$ & $\ldots$ & $\mathrm{C} 2 \mathrm{IH} 29 \mathrm{~N} 3 \mathrm{~S}$ & |400742-46-2 \\
\hline PTI-2 & & ${ }_{-0}=\beth^{N<}$ & Pentyl & $\mathrm{H}$ & $\ldots$ & $\mathrm{C} 23 \mathrm{H} 33 \mathrm{~N} 3 \mathrm{OS}$ & Not attributed \\
\hline \multicolumn{8}{|l|}{ Naphthylmethyl derivatives } \\
\hline JWHI75 & & & Pentyl & $\mathrm{H}$ & $\ldots$ & $\mathrm{C} 24 \mathrm{H} 25 \mathrm{~N}$ & $619294-35-8$ \\
\hline
\end{tabular}

Note: “..." demonstrates that there is no substituant on the phenyl core in position R' or R3.

Abbreviation: CAS, Chemical Abstracts Service.

different and various chemical families. The major structural group is the indole group (Table 1, Figure 1) which includes several indole sub-groups (R): benzoyl, Naphthol, phenylacetyl, alkyl, piperazinyl, carboxylate, carboxamide, thiazolyl, and naphthylmethyl derivatives.

Besides these abundant indole derivatives, many other SCs groups have very different structural characteristics (Table 2). In spite of the diversity of products, some similarities should be noted: 1) a quite constant unsaturated and substituted five-membered ring incorporating at least one nitrogen (pyrrole), and merged to another aromatic cycle; and 2) within series, changes in substituent are often limited to the simple addition of a methyl or halogen group to a linear alkyl chain. Nevertheless, more marked modifications are possible, making the classification of such compounds very difficult. Some of these compounds are chiral, and can exist in two stereoisomer forms. SCs are usually referred to by usual trade names such as JWH-XXX (John W Huffmann), CP-XX, XXX (Charles Pfizer), HU-XXX (Hebrew University), AMXXXX (Alexandros Makriyannis), and many others. Note that within a trade name family, several chemical classes may be represented. For example, JWH-XXX series includes Naphthol indoles, phenylacetyl indoles, naphthylmethyl indoles, naphthylmethyl indene, and Naphthol pyrroles.

\section{Current and developing analytical methods for detection Immunochemistry}

As a general rule, immunoassay screening methodologies used to detect cannabis fail to detect SCs, but some specific 
Table 2 Chemical structure of other cannabinoid derivatives

\begin{tabular}{|c|c|c|c|c|c|c|c|c|}
\hline \multicolumn{9}{|l|}{ Indene derivatives } \\
\hline Name & $\mathbf{R}$ & $\mathbf{R}^{\prime}$ & $\mathbf{R I}$ & $\mathbf{R 2}$ & R3 & R4 & Formula & CAS number \\
\hline \multicolumn{9}{|l|}{ Naphthylmethylindenes } \\
\hline JWH-I76 & $T_{R}+20$ & $\mathrm{H}$ & Pentyl & $\ldots$ & & & $\mathrm{C} 25 \mathrm{H} 24$ & $619297-62-1$ \\
\hline JWH-220 & & Methyl & Pentyl & $\ldots$ & & & $\mathrm{C} 26 \mathrm{H} 26$ & Not attributed \\
\hline \multicolumn{9}{|l|}{ Pyrrole derivatives } \\
\hline \multicolumn{9}{|l|}{$\operatorname{Rin}^{R 2}-\pi T^{R}$} \\
\hline \multicolumn{9}{|l|}{ Naphthoyl pyrroles } \\
\hline JWH-030 & ing & $\ldots$ & Pentyl & $\ldots$ & & & $\mathrm{C} 2 \mathrm{OH} 2 \mathrm{INO}$ & 162934-73-8 \\
\hline JWH-I45 & & $\ldots$ & Pentyl & Phenyl & & & $\mathrm{C} 26 \mathrm{H} 25 \mathrm{NO}$ & $914458-19-8$ \\
\hline JWH-I47 & & $\ldots$ & Hexyl & Phenyl & & & $\mathrm{C} 27 \mathrm{H} 27 \mathrm{NO}$ & $9 \mid 4458-20-1$ \\
\hline JWH-307 & & $\ldots$ & Pentyl & 2-fluorophenyl & & & $\mathrm{C} 26 \mathrm{H} 24 \mathrm{FNO}$ & $914458-26-7$ \\
\hline JWH-368 & & $\ldots$ & Pentyl & 3-fluorophenyl & & & $\mathrm{C} 26 \mathrm{H} 24 \mathrm{FNO}$ & $9 \mid 4458-31-4$ \\
\hline JWH-370 & & $\ldots$ & Pentyl & 2-methylphenyl & & & $\mathrm{C} 27 \mathrm{H} 27 \mathrm{NO}$ & $914458-22-3$ \\
\hline \multicolumn{9}{|l|}{ Indazole derivatives } \\
\hline \multicolumn{9}{|c|}{$\overbrace{N_{N}}^{R}$} \\
\hline \multicolumn{9}{|c|}{ Carboxamide indazole derivatives } \\
\hline AB-CHMINACA & ${\stackrel{\rho}{N^{-}}-R^{\prime}}^{2}$ & & o & $\ldots$ & & & $\mathrm{C} 2 \mathrm{OH} 28 \mathrm{~N} 4 \mathrm{O} 2$ & || $85887-2|-|$ \\
\hline AB-FUBINACA & & & $-\infty$ & $\ldots$ & & & $\mathrm{C} 2 \mathrm{OH} 2 \mathrm{IFN} 4 \mathrm{O} 2$ & || $85282-0 \mid-2$ \\
\hline AB-PINACA & & & Pentyl & $\ldots$ & & & $\mathrm{Cl} 8 \mathrm{H} 26 \mathrm{~N} 4 \mathrm{O} 2$ & |445752-09-9 \\
\hline 5F-AB-PINACA & & & 5-fluoropentyl & $\ldots$ & & & $\mathrm{Cl} 8 \mathrm{H} 25 \mathrm{FN} 4 \mathrm{O} 2$ & Not attributed \\
\hline ADB-CHMINACA = & & & 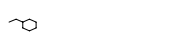 & $\cdots$ & & & $\mathrm{C} 2 \mathrm{IH} 30 \mathrm{~N} 4 \mathrm{O} 2$ & $8322231-92-1$ \\
\hline \multicolumn{9}{|l|}{ MAB-CHMINACA } \\
\hline ADB-FUBINACA & & & $-a$ & $\ldots$ & & & $\mathrm{C} 2 \mathrm{IH} 23 \mathrm{FN} 4 \mathrm{O} 2$ & $|445583-5|-6$ \\
\hline ADB-PINACA & & & Pentyl & $\ldots$ & & & $\mathrm{Cl} 9 \mathrm{H} 28 \mathrm{~N} 4 \mathrm{O} 2$ & $1633766-73-0$ \\
\hline 5F-ADB-PINACA & & & 5 -fluoropentyl & $\ldots$ & & & $\mathrm{Cl} 19 \mathrm{H} 27 \mathrm{FN} 4 \mathrm{O} 2$ & Not attributed \\
\hline $5 F-A D B$ & & $\gamma_{\mathrm{NH}_{2}}$ & 5 -fluoropentyl & $\ldots$ & & & $\mathrm{C} 2 \mathrm{OH} 28 \mathrm{FN} 3 \mathrm{O} 3$ & Not attributed \\
\hline $5 F-A M B=5 F-A M P$ & & & 5 -fluoropentyl & $\ldots$ & & & $\mathrm{Cl} 19 \mathrm{H} 26 \mathrm{FN} 3 \mathrm{O} 3$ & Not attributed \\
\hline APINACA $=$ AKB-48 & & & Pentyl & $\ldots$ & & & $\mathrm{C} 23 \mathrm{H} 3 \mathrm{IN} 3 \mathrm{O}$ & I345973-53-6 \\
\hline 5F-APINACA = 5F-AKB-48 & & xio- & 5 -fluoropentyl & $\cdots$ & & & $\mathrm{C} 23 \mathrm{H} 30 \mathrm{FN} 3 \mathrm{O}$ & $1400742-13-3$ \\
\hline FUB-APINACA = FUB-AKB-48 & & & $\infty$ & $\ldots$ & & & $\mathrm{C} 25 \mathrm{H} 26 \mathrm{FN} 3 \mathrm{O}$ & Not attributed \\
\hline 5F-APP-PINACA = FU-PX = PX-2 & & $\notin$ & 5 -fluoropentyl & $\cdots$ & & & $\mathrm{C} 22 \mathrm{H} 25 \mathrm{FN} 4 \mathrm{O} 2$ & Not attributed \\
\hline CUMYL-PINACA & & & Pentyl & $\ldots$ & & & $\mathrm{C} 22 \mathrm{H} 27 \mathrm{~N} 3 \mathrm{O}$ & Not attributed \\
\hline CUMYL-5FPINACA = SGT-25 & & $x$ & 5 -fluoropentyl & $\ldots$ & & & $\mathrm{C} 22 \mathrm{H} 26 \mathrm{FN} 3 \mathrm{O}$ & Not attributed \\
\hline CUMYL-THPINACA & & $x_{0}$ & $\chi_{0}$ & $\ldots$ & & & $\mathrm{C} 23 \mathrm{H} 27 \mathrm{~N} 3 \mathrm{O} 2$ & Not attributed \\
\hline $\mathrm{MN}-18$ & & & Pentyl & $\ldots$ & & & $\mathrm{C} 23 \mathrm{H} 23 \mathrm{~N} 3 \mathrm{O}$ & $|39| 484-80-2$ \\
\hline $5 F-M N-18$ & & $\infty$ & 5-fluoropentyl & $\ldots$ & & & $\mathrm{C} 23 \mathrm{H} 22 \mathrm{FN} 3 \mathrm{O}$ & |44558|-9|-8 \\
\hline \multicolumn{9}{|l|}{ Carboxylate indazole derivatives } \\
\hline NPB-22 & 只 $_{0}-R^{\prime}$ & & Pentyl & $\ldots$ & & & $\mathrm{C} 22 \mathrm{H} 2 \mathrm{IN} 3 \mathrm{O} 2$ & $|445579-6|-2$ \\
\hline 5F-NPB-22 & & & 5 -fluoropentyl & $\ldots$ & & & $\mathrm{C} 22 \mathrm{H} 2 \mathrm{FFN} 3 \mathrm{O} 2$ & | 4455579-79-2 \\
\hline FUB-NPB-22 & & $\downarrow^{N}$ & $\infty$ & $\ldots$ & & & $\mathrm{C} 24 \mathrm{HI} 6 \mathrm{FN} 3 \mathrm{O} 2$ & Not attributed \\
\hline SDB-005 & & & Pentyl & $\ldots$ & & & $\mathrm{C} 23 \mathrm{H} 22 \mathrm{~N} 2 \mathrm{O} 2$ & Not attributed \\
\hline 5F-SDB-005 & & $\infty$ & 5-fluoropentyl & $\ldots$ & & & $\mathrm{C} 23 \mathrm{H} 2 \mathrm{IFN} 2 \mathrm{O} 2$ & Not attributed \\
\hline
\end{tabular}

Benzimidazole derivatives

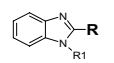

FUBIMINA = BIM-220I

Dihydroisoindole-I one derivatives

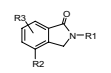

JTE 7-3I

Pentylamino

Methoxy

$\mathrm{C} 22 \mathrm{H} 28 \mathrm{~N} 2 \mathrm{O} 3$

194358-72-0

(Continued) 
Table 2 (Continued)

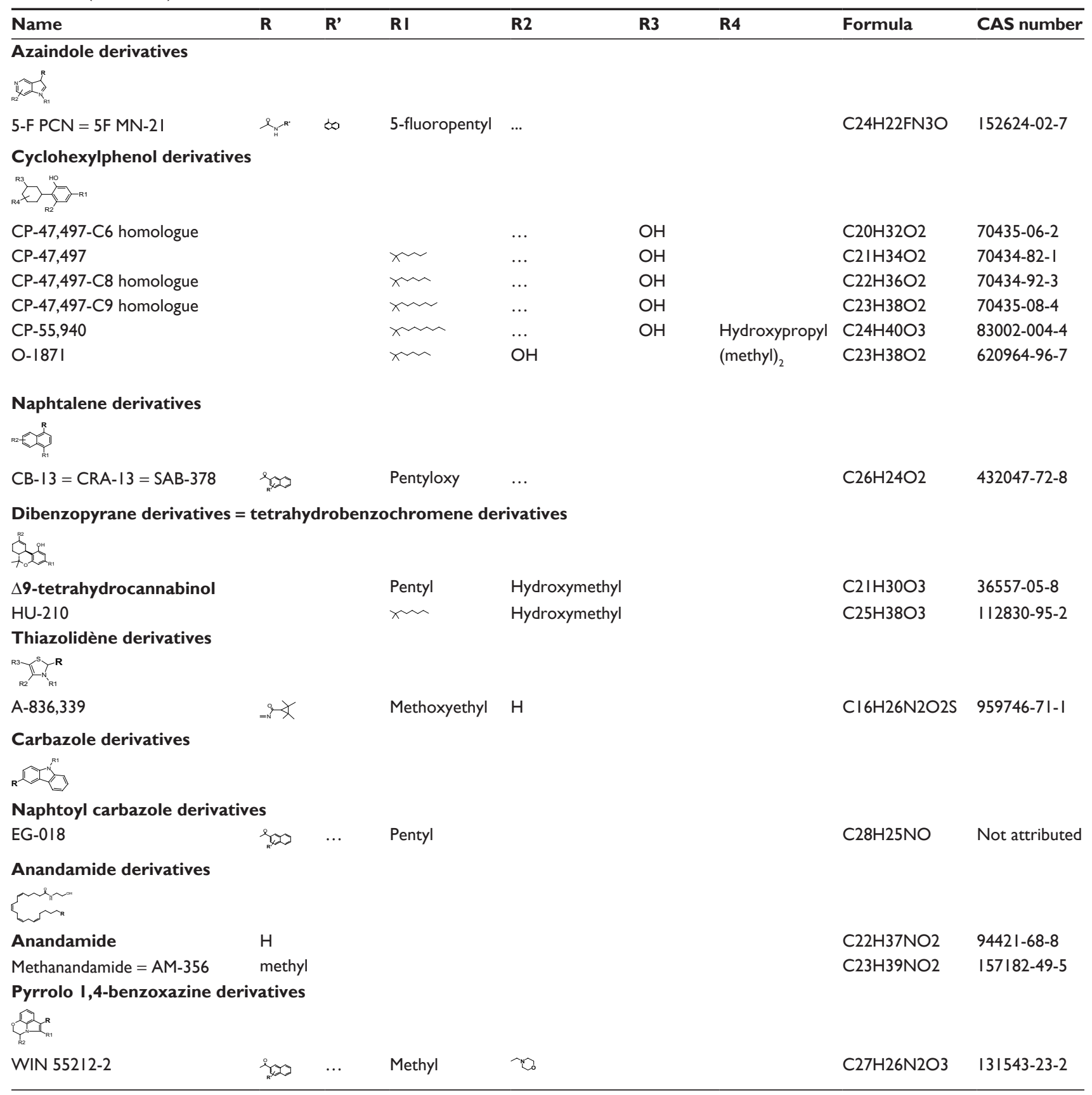

Notes: “..." demonstrates that there is no substituant on the aromatic core in position R' or R2. When there is no R3 or R4 in the basic structure the column was left blank. " $\mathrm{H}$ " has been included when the structure was not a phenyl or an aromatic core.

Abbreviation: CAS, Chemical Abstracts Service.

enzyme-linked immunosorbent assays or homogenous enzyme immunoassays have been recently designed to detect the use of common SCs in urine, such as JWH-018, JWH250, UR-144, and others. ${ }^{24-26}$

\section{Gas chromatography-mass spectrometry}

Several gas liquid chromatography (LC) connected to electron-impact mass spectrometry (GC-MS) methods have been developed to rapidly identify and quantify SCs in herbal and powder materials. ${ }^{27-31}$ In our own experience we used a DB-5 fused-silica capillary column (30 $\mathrm{m} \times 0.25$ $\mathrm{mm} \times 0.25 \mu \mathrm{m})$. Oven temperature was increased from $100^{\circ} \mathrm{C}$ (hold time: 1 minute) by $8^{\circ} \mathrm{C} / \mathrm{min}$ up to $280^{\circ} \mathrm{C}$ (26.5 minutes), with helium flow at $1 \mathrm{~mL} / \mathrm{min}$.

A standard method to screen new psychoactive substances was to have a total runtime of 60 minutes, with the retention times of SCs ranging between 21.9 and 48.0 minutes. The rank order (retention time) of ten analyzed SCs was as follows 


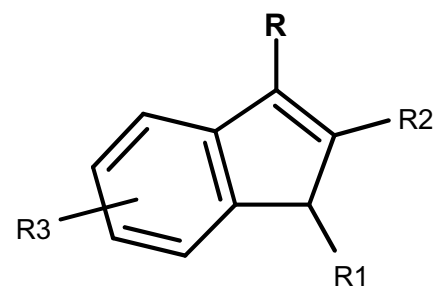

Figure I Chemical structure of indole derivatives.

from the lowest rank to the highest rank: UR-144; JWH-250; HU210; RCS-4; JWH-073; JWH-018; JWH-019; AM-2201; JWH-122; JWH-081; and JWH-200. The selected mass spectrometry (MS) data that we collected in the literature, and from our own data bank are summarized in Table 3, and identify several SCs, some are among the most notorious. Consistent with the large variety of chemical structures, mass spectra under electron impact conditions differ largely from one $\mathrm{SC}$ to another, with some discrepancies between reported spectra in terms of ion intensity. An intense molecular ion $\left(\mathrm{M}^{+}\right)$is frequently present and fragmentations are commonly observed on both sides of the carbonyl group: for example, SCs bearing an n-pentyl moiety show an intense ion at $\mathrm{m} / \mathrm{z}=214$ corresponding to $n$-pentyl indoloyl, ${ }^{27}$ and those bearing a 5-fluoropentyl moiety show an intense ion at $\mathrm{m} / \mathrm{z}$ $=232$ (fluoropentyl indoloyl). The mass-spectral fragmentation pattern of SCs also includes formations of different immonium ions at $\mathrm{m} / \mathrm{z}=144$ (indoloyl moiety), 155 (Naphthol moiety), or 127 (Naphthol moiety). ${ }^{32}$ GC-MS methods have been developed to determine the composition of Spice herbal mixtures but appear inadequate to measure concentrations in the user's biological specimen.

\section{LC-MS}

Different LC-MS/MS screening or semi-quantitative and quantitative methods have been published and identify and quantify SCs in various specimens - urine, serum, blood, oral fluid, or hair. ${ }^{33-40} \mathrm{~A}$ classic or ultra-performing reverse phase $\mathrm{C} 18$ is typically used for the chromatographic separation prior to analysis by LC-tandem MS using electrospray positive ionization multiple reaction monitoring mode. ${ }^{33-40}$ Multiple reaction monitoring transitions for SCs are presented in Table 4. For all substances, the dominant Q1 ions were the protonated molecular ions $\left([\mathrm{M}+\mathrm{H}]^{+}\right)$, and at least two product ions of acceptable abundance can be obtained. Ions $\mathrm{C} 11 \mathrm{H}_{7} \mathrm{O}^{+}$ $(\mathrm{m} / \mathrm{z}=155.0)$ and $\mathrm{C} 10 \mathrm{H} 7^{+}(\mathrm{m} / \mathrm{z}=127.0)$ can be referred to as common characteristic ions. Simultaneous determinations and quantifications of SCs metabolites have also been successfully applied to urine specimens. ${ }^{37,41}$ In order to complete targeted MS/MS screening, ultra-high performance LC coupled with high resolution quadrupole time-of-flight MS high-sensitivity methods have also been developed to directly screen SCs in specimens such as in urine or hair. ${ }^{42-45}$ Real time MS was employed to detect the SCs protonated molecules $[\mathrm{M}+\mathrm{H}]^{+}$ directly on herbal matrices, without extraction or sample preparation. ${ }^{46}$ The limits of quantification for the assay of SCs in urine, using an electrospray ionization source in positive polarity, are approximately $0.1-1 \mu \mathrm{g} / \mathrm{L}$, with upper limits of linearity at $50-100 \mu \mathrm{g} / \mathrm{L} .{ }^{37}$ Accordingly, LC-MS-MS is the only method that presents sufficient sensibility to quantify SCs in biological fluids.

\section{Extraction procedures for quantification in herbal and biological samples}

Herbal material has to be crushed and stirred or sonicated in methanol or ethanol before chromatographic analysis. Human urine is the favorite biological matrix explored to detect SCs consumption. Biological samples may be enzymatically hydrolyzed before pretreatment. Solid-phase extraction or simple precipitation is next used for oral fluid or urine samples. ${ }^{33,37,47}$ Basic liquid-liquid extraction after deproteinization with acetonitrile followed by a concentration step is generally preferred to prepare blood samples. . $^{34,38}$ Liquid-liquid extraction is preceded by an incubation with $\mathrm{NaOH}$ at $95^{\circ} \mathrm{C}$ for finely cut hair samples.

\section{Experimental pharmacology and neuropharmacology}

By acting as retrograde messengers at various synapses, endocannabinoids (anandamide and 2-arachidonoylglycerol) have a neuromodulatory role in motor activity, pain perception, feeding, emotional state, learning and memory, and reward behaviors. ${ }^{48,49}$ They also influence cardiovascular and immune systems and control progenitor cell proliferation. ${ }^{48}$ Endocannabinoids bind to $\mathrm{CB} 1$ receptors mainly located at the terminals of central and peripheral neurons and to CB2 receptors principally expressed in immune and hematopoietic cells both within and outside the central nervous system. CB1 receptors are often localized presynaptically where their stimulation usually inhibits neurotransmitter release and accounts for most of the neurobehavioral effects, while CB2 receptors are mainly implicated in the immunomodulatory effects. Yet, $\mathrm{CB} 1$ receptors are also present at much lower concentrations in peripheral tissues and CB2 in neurons and microglia.

\section{In vitro}

The complex molecular architecture of the cannabinoid receptors allow for a single receptor to recognize multiple 
Table 3 Gas chromatography mass spectrometry (GC-MS) identification of synthetic cannabinoids

\begin{tabular}{|c|c|c|c|c|c|}
\hline \multirow[t]{2}{*}{ Chemical class } & \multirow[t]{2}{*}{ Name } & \multirow[t]{2}{*}{ Molecular weight } & \multicolumn{2}{|c|}{ GC-MS identification (electronic impact) } & \multirow[t]{2}{*}{ Reference } \\
\hline & & & Base peak & Other peaks & \\
\hline \multirow[t]{7}{*}{ Benzoyl indoles } & AM-694 & 435,27 & 232 & $435,220,380$ & 30 \\
\hline & & & 232 & $435,220,360$ & 28 \\
\hline & RCS-4 & $32 I, 4 I$ & 312 & $284,214,135$ & 30 \\
\hline & & & 321 & $135,264,214$ & 27 \\
\hline & & & 321 & $265,135,214$ & Personal data \\
\hline & RCS-4-(N-Me) & 265,31 & 158 & $265,77,264,266$ & 27 \\
\hline & WIN-48,098 & 378,46 & 100 & 135,378 & 28 \\
\hline \multirow[t]{31}{*}{ Naphthoyl indoles } & JWH-0I8 & $34 I, 45$ & 341 & $284,214,127,324$ & 30 \\
\hline & & & $34 I$ & $284,214,127,324$ & 28 \\
\hline & & & $34 I$ & $325,285,215$ & Personal data \\
\hline & JWH-0I9 & 355,47 & 355 & $284,228,127,338$ & 30 \\
\hline & & & 355 & $338,284,228$ & Personal data \\
\hline & JWH-073 & 327,42 & 327 & $200,284,127,310$ & 30 \\
\hline & & & 327 & $200,284,310,127$ & 28 \\
\hline & & & 327 & $310,284,200$ & Personal data \\
\hline & JWH-08I & $37 I, 47$ & 371 & $354,3|4,2| 4,185$ & 30 \\
\hline & & & 371 & $354,370,314,214,185$ & 27 \\
\hline & & & 371 & $354,3|4,2| 4$ & Personal data \\
\hline & JWH-I22 & 355,47 & 355 & $338,298,215$ & 30 \\
\hline & & & 355 & $298,214,338$ & Personal data \\
\hline & JWH-122-pentenyl & 353,45 & 353 & $351,335,127,284$ & 31 \\
\hline & JWH-200 & 384,47 & 100 & $384,339,155,127$ & Personal data \\
\hline & JWH-2I0 & 369,50 & 369 & $352,312,214,340$ & 30 \\
\hline & & & 369 & $352,3|2,2| 4,368,340$ & 27 \\
\hline & JWH-4I2 & 359,44 & 359 & $302,145,173,214$ & 29 \\
\hline & AM- 1220 & 382,21 & 98 & $70,127,254,284$ & 31 \\
\hline & & & 98 & $70,127,155,254$ & 32 \\
\hline & AM-1220- azepane & 382,21 & 382 & $84,127,184,57$ & 31 \\
\hline & & & 127 & $84,57,382,98$ & 32 \\
\hline & AM-220I & 359,44 & 359 & $284,232,342,358,127$ & 31 \\
\hline & & & 359 & $127,284,232,342$ & 29 \\
\hline & & & 359 & $127,284,232,342$ & 28 \\
\hline & & & 359 & $284,232,342,358$ & 27 \\
\hline & & & 359 & $284,232,127,342$ & 30 \\
\hline & & & 355 & $338,284,228$ & Personal data \\
\hline & AM-220I-pMe & 373,46 & 373 & $298,232,356,372$ & 31 \\
\hline & & & 373 & $298,232,356,372$ & 27 \\
\hline & AM-2232 & 352,43 & 352 & $351,335,225,127$ & 31 \\
\hline \multirow[t]{4}{*}{ Phenylacetyl indoles } & JWH-203 & 339,86 & 214 & $144,116,339$ & 30 \\
\hline & JWH-250 & 335,44 & 214 & $144,116,335$ & 30 \\
\hline & & & 214 & $144,335,116$ & 28 \\
\hline & & & 214 & $144,215,335$ & Personal data \\
\hline \multirow[t]{3}{*}{ Alkoyl indoles } & UR-I44 & 311,2 & 214 & $|44,296,215,31|$ & 31 \\
\hline & & & 215 & $296,144,311$ & Personal data \\
\hline & XLR-I I & 329,2 & 232 & $|44,233,329,4|$ & 31 \\
\hline \multirow[t]{2}{*}{ Carboxylate indoles } & $\mathrm{MN}-27$ & 396 & 109 & 252,396 & 128 \\
\hline & NM-220I & 375,16 & 232 & 144,115 & 128 \\
\hline Carboxamide indoles & STS-I35 & 382,2 & 232 & $382,307,144,383$ & 31 \\
\hline \multirow[t]{2}{*}{ Naphtoyl pyrroles } & JWH-I47 & & 381 & $155,127,310,296$ & 30 \\
\hline & FUB-NPB-22 & 397,4 & 109 & 253,397 & 128 \\
\hline \multirow[t]{2}{*}{ Carboxylate indazoles } & 5-fluoro-NPB-22 & 377,4 & 233 & 145,377 & 128 \\
\hline & 5-fluoro-SDB-005 & 376 & 233 & $145,213,376$ & 128 \\
\hline Dibenzopyranes & HU-2IO & 386,57 & 303 & 387,33 I, 285, 270 & Personal data \\
\hline
\end{tabular}


Table 4 Liquid chromatography - tandem mass spectrometry identification of synthetic cannabinoids

\begin{tabular}{|c|c|c|c|c|}
\hline Name & $\begin{array}{l}\text { Molecular } \\
\text { weight }\end{array}$ & $\begin{array}{l}\text { Precursor } \\
\text { ion }\end{array}$ & Product ions & Reference \\
\hline \multicolumn{5}{|c|}{ Positive mode } \\
\hline AM-25I & 555.2 & 555.0 & $454.0 ; 472.0$ & 39 \\
\hline AM-694 & 435.3 & 435.9 & 231,$0 ; 309,2 ; 203,2$ & 39 \\
\hline AM-220I & 359.4 & 360.1 & I55.I; 127.2 & II,34,37,40 \\
\hline AM- 1220 & 382.5 & 383,2 & II2,0; 98,I; 286,2 & 34,36 \\
\hline AM-I24I & 503.3 & 504.1 & $98.0 ; 275.0$ & 39 \\
\hline AM-220I & 359.4 & 360.2 & $155.0 ; 232.0$ & 39 \\
\hline AM-2233 & 458.3 & 459.1 & II2.I; $98.1 ; 230.0$ & 33,39 \\
\hline $\mathrm{HU}-210$ & 386.6 & 387,2 & 243,$2 ; 261,3 ; 85,0$ & 36,39 \\
\hline JWH-007 & 355.5 & 356.2 & $155.2 ; 127.2$ & $33,38,39$ \\
\hline JWH-OII & 383.5 & 384.2 & $155.0 ; 286.0$ & 39 \\
\hline $\mathrm{JWH}-0 \mathrm{I5}$ & 327.4 & 328.1 & I55.I; 200.I; 127.0 & $34,36,38$ \\
\hline JWH-0I8 & 341.4 & 342.1 & $155.1 ; 214.2 ; 127.1$ & $36-38,40$ \\
\hline JWH-0I9 & 355.5 & 356.2 & I55.I; I26.9; 228.I & $34,36,37$ \\
\hline JWH-020 & 369.5 & 370.4 & $155.1 ; 242.1 ; 127.1$ & 33,36 \\
\hline JWH-022 & 339.4 & 340.2 & $155.0 ; 212.0$ & 39 \\
\hline JWH-030 & 291.4 & 292.2 & $155.0 ; 168.0$ & 39 \\
\hline JWH-073 & 327.4 & 328.1 & 155,$0 ; 200,0 ; 127,0$ & $34,36-38,40$ \\
\hline JWH-08I & 371.5 & 372.2 & $185.1 ; 157.1 ; 127.0$ & $34,36,37$ \\
\hline JWH-098 & 385.5 & 386.2 & I85.0; 198.0; 228.0 & 39 \\
\hline JWH-I22 & 355.5 & 356.1 & $169.1 ; 214.2 ; 141.0$ & $34,36-38,40$ \\
\hline JWH-I82 & 383.5 & 384.2 & 197.0; 214.0; 144 & 38,39 \\
\hline JWH-200 & 384.5 & 385.2 & $155.1 ; 114.0 ; 127.0$ & $34,36,37$ \\
\hline JWH-20I & 335.4 & 336.2 & I2I.0; 135.0; 214.0 & 39 \\
\hline JWH-203 & 339.9 & $340, I$ & |24.9; I88.I; 89.0 & 33,36 \\
\hline $\mathrm{JWH}-210$ & 369.5 & 370.1 & $183.1 ; 214.1 ; 153.1$ & $36-38$ \\
\hline JWH-249 & 384.3 & 385 & 144 & 38 \\
\hline JWH-250 & 335.4 & 336.1 & I2I.2; 9|.I; 200.3 & $34,36-38$ \\
\hline JWH-25I & 319.4 & 320,1 & 104,$9 ; 214,1 ; 144,2$ & $33,34,36,37$ \\
\hline JWH-302 & 335.4 & 336 & $144 ; 12 \mid$ & 38 \\
\hline JWH-307 & 385.5 & 386,2 & I55,0; I27,I; 77,I & $33,34,36$ \\
\hline JWH-398 & 375.9 & 376.1 & $189 ; 161.1 ; 126.1$ & $33,36,37$ \\
\hline $\mathrm{JWH}-412$ & 359.4 & 360.4 & $173.2 ; 145.1$ & 33 \\
\hline JWH-424 & 420.3 & 422 & 144 & 38 \\
\hline MAM-220I & 373.5 & 374.1 & $169.0 ; 115.0$ & 37,40 \\
\hline $\mathrm{RCS}-4$ & 321.4 & 322,2 & 135,$0 ; 77,0 ; 92,0$ & 36,37 \\
\hline RCS-8 & 375.5 & 376,2 & 121,$0 ; 90,9 ; 143,9$ & 36,37 \\
\hline WIN-48,098 & 378.5 & 379,1 & 134,$9 ;|| 4,0 ; 77, \mid$ & 36 \\
\hline WIN 55,2I2-2 & 426.5 & 427,3 & 155,$1 ; 127,0 ; 100,0$ & $33,36,38$ \\
\hline XRL-II & 329.5 & 330.1 & $125.1 ; 232.0$ & 37 \\
\hline \multicolumn{5}{|c|}{ Negative mode } \\
\hline CP-47,497-C7 & 318.5 & 317.1 & $245.1 ; 159.1$ & 37 \\
\hline CP-47,497-C8 & 332.5 & 331.1 & $259.1 ; 159.0$ & 37 \\
\hline HU-2IO & 386.6 & 385.6 & $301.3 ; 281.1$ & 37 \\
\hline
\end{tabular}

classes of compounds. ${ }^{50} \mathrm{SCs}$ which present a large variety of chemical structures like that previously shown in Table 1 and 2 , bind to the two types of cannabinoid receptors with a varying degree of affinity. These $\mathrm{CB} 1$ and $\mathrm{CB} 2$ receptor affinities of SCs have been determined in displacement assays using tritiated cannabinoid receptor ligands and membranes obtained from brain (CB1-rich), spleen (CB2rich), or using culture cells transfected with $\mathrm{CB} 1$ or $\mathrm{CB} 2$ receptors. ${ }^{51} \mathrm{Ki}$ values of SCs collected from literature are grouped in Table 5.7,52-64 The majority of compounds used as drug of abuse have $\mathrm{Ki}$ in the range 1 to $10 \mathrm{nM}$ or 10 to $100 \mathrm{nM}$ for both $\mathrm{CB} 1$ and $\mathrm{CB} 2$ receptors. Some synthetic compounds bind more strongly to $\mathrm{CB} 1$ receptor than $\Delta^{9}$-THC. JWH-210 from the Naphthoylindole family, acts as a potent cannabinoid agonist with both the $\mathrm{CB} 1$ and $\mathrm{CB} 2$ receptors, with $\mathrm{Ki}$ values of $0.46 \mathrm{nM}$ at $\mathrm{CB} 1$ and $0.69 \mathrm{nM}$ at $\mathrm{CB} 2 .{ }^{52}$ At the opposite end, JWH-071 binds with the central CB1 receptor $(\mathrm{Ki}=1,340 \mathrm{nM})$ and the peripheral $\mathrm{CB} 2$ receptor 
Table 5 Affinities of much of synthetic cannabinoids

\begin{tabular}{|c|c|c|c|c|c|}
\hline & \multicolumn{5}{|l|}{ CBI } \\
\hline & $\mathbf{K i}<\mathbf{I} \mathbf{n M}$ & $\mathrm{I} \mathbf{n M}<\mathrm{Ki}<10 \mathrm{nM}$ & $10 \mathrm{nM}<\mathrm{Ki}<100 \mathrm{nM}$ & $\begin{array}{l}100 \mathrm{nM}<\mathrm{Ki}< \\
1000 \mathrm{nM}\end{array}$ & $\mathrm{Ki}>1000 \mathrm{nM}$ \\
\hline \multicolumn{6}{|l|}{$\overline{\text { CB2 }}$} \\
\hline \multirow[t]{2}{*}{$\mathbf{K i}<\mathbf{I} \mathbf{n M}$} & $\mathrm{JWH}-210^{52^{*}}$ & JWH-149;52 AB-005;56 & AM- $|22|^{54}$ & A- $836,339^{57}$ & \\
\hline & & A- 834,$735 ; ;^{56}$ O- $1871^{64}$ & & & \\
\hline \multirow[t]{7}{*}{$\mathbf{I} \mathbf{n M}<\mathbf{K i}<10 \mathrm{nM}$} & AM-694; ${ }^{54} \mathrm{JWH}-122 ; ;^{53}$ & AM-2233; $;^{58} \mathrm{JWH}-007 ; 7,52$ & JWH-424; ${ }^{61}$ UR-144;56 & & \\
\hline & JWH-I82;52 AM-2232 54 & JWH-018;53 JWH-019;53 & JWH- I45;52 JWH- I47;52 & A- $796,260^{56}$ & \\
\hline & & JWH-098; ${ }^{53} \mathrm{JWH}-387 ;{ }^{59}$ & $\mathrm{JWH}-368^{52}$ & & \\
\hline & & JWH-398; ${ }^{59} \mathrm{JWH}-412 ;{ }^{59}$ & & & \\
\hline & & AM-220; ${ }^{57} \mathrm{JWH}-203 ;{ }^{55}$ & & & \\
\hline & & JWH-250;55 NM-2201; ${ }^{54}$ & & & \\
\hline & & JWH-307;52 JWH-370;52 & & & \\
\hline \multirow[t]{4}{*}{$10 \mathrm{nM}<\mathrm{Ki}<100 \mathrm{nM}$} & & JWH-073; ${ }^{53} \mathrm{JWH}-081 ;^{53}$ & AM-679; ${ }^{54} \Delta$ 9-THC; $; 2$ & JWH-0I5;53 & \\
\hline & & AM-I $235 ; ;^{54} \mathrm{JWH}-249^{55}$ & JWH-204;,55 JWH-253;55 & JWH-25I;,55 & \\
\hline & & & JWH-302;55 JWH-311;55 & $\mathrm{MN}-25^{62}$ & \\
\hline & & & JWH-I76;63 JWH-22063 & & \\
\hline \multirow[t]{2}{*}{$100 \mathrm{nM}<\mathrm{Ki}<1000 \mathrm{nM}$} & & & JWH-167;55 & JWH-206;55 & $\mathrm{JWH}-201 ;{ }^{55} \mathrm{JWH}-316^{55}$ \\
\hline & & & methanandamide ${ }^{60}$ & $\mathrm{JWH}-208^{55}$ & \\
\hline $\mathrm{Ki}>1000 \mathrm{nM}$ & & & & JWH-20955 & JWH-071; ${ }^{53} \mathrm{JWH}-207^{55}$ \\
\hline
\end{tabular}

Notes: *The number in exponent is the reference in the literature giving the Ki. The bold text represents the lead natural cannabinoid of Cannabis sativa.

$(\mathrm{Ki}=2,940 \mathrm{nM})$ at a micromolar level. ${ }^{53}$ The majority of SCs are unspecific $\mathrm{CB} 1 / \mathrm{CB} 2$ ligands (Table 5), with only a few are $\mathrm{CB} 2$ selective such as $\mathrm{AM}-1221(\mathrm{Ki}=0.28 \mathrm{nM}$ at $\mathrm{CB} 2$ vs $52.3 \mathrm{nM}$ at the $\mathrm{CB} 1)^{54}$ and $\mathrm{A}-836,339(\mathrm{Ki}=0.64 \mathrm{nM}$ at $\mathrm{CB} 2$ vs $270 \mathrm{nM}$ at $\mathrm{CB} 1)^{57}$

$\mathrm{CB} 1$ and $\mathrm{CB} 2$ receptors are G protein-coupled receptors. Two in vitro assays using cell membranes or cultured cells that express $\mathrm{CB} 1$ or $\mathrm{CB} 2$ receptors are commonly employed to determine the SCs agonist property; the $\left[{ }^{35} \mathrm{~S}\right]$ GTP $\gamma \mathrm{S}$ assay that measures cannabinoid receptor agoniststimulated binding to $\mathrm{G}$ proteins, and the cyclic adenosine monophosphate assay that quantitatively determine the drug-induced production of this important second messenger. ${ }^{51}$

As a general rule, the agonists show little selectivity between the $\mathrm{CB} 1$ and $\mathrm{CB} 2$ receptors, while the antagonist compounds are highly selective. ${ }^{65}$

\section{In vivo}

SCs that target $\mathrm{CB} 1$ and/or CB2 receptors may be classified in $\mathrm{CB} 1 / \mathrm{CB} 2$ agonists, $\mathrm{CB} 2$ selective agonists, peripherally restricted $\mathrm{CB} 1 / \mathrm{CB} 2$ agonists, $\mathrm{CB} 1 / \mathrm{CB} 2$ antagonists, and inverse agonists. ${ }^{66}$ Additionally, chiral centers can exist in many SCs and stereoisomer forms may differ in their pharmacological potencies. ${ }^{67}$ All may have an interest as recreational or medicinal drugs. In vivo in mice, activation of CB1 receptors produces a "tetrad" of dose-dependent effects, including suppression of locomotor activity, hypo- thermia, immobility in the ring test, and antinociception in the tail-flick or hot-plate test. ${ }^{66}$ This cannabinoid tetrad is extremely useful in the characterization of the biological activity of SCs but the development of CB1, CB2, or CB1/ $\mathrm{CB} 2$ knockout mice provided additional methods to test the SCs specificities. In addition, agonistic binding to CB1 receptors resulted in behavioral effects including euphoria, elevation or anxiety, or alteration of memory ${ }^{68}$ In agreement with in vitro studies, in vivo studies show SCs pharmacological effects commonly more potent than $\Delta^{9}$-THC. Similarly, SCs perfectly substituted for $\Delta^{9}$-THC in discrimination studies. ${ }^{3}$ Activation of $\mathrm{CB} 2$ receptors results in release of immunomodulating agents and reduction of inflammatory induced pain. Accordingly, our purpose primarily focused on specific or unspecific $\mathrm{CB} 1$ agonists.

Many studies in mice suggest a major regulatory role of cannabinoid signaling in pregnancy with multiple sites and stages of pregnancy potential targets of SCs, including preimplantation embryo development, oviductal embryo transport, implantation, placentation, and parturition. ${ }^{69}$

Studies on animals have proved that cannabinoids have an addictive potential, involving reward system and CB1 receptors. $\Delta^{9}$-THC activates the brain reward circuit by stimulating the mesolimbic dopamine system. ${ }^{70,71}$ It has been previously demonstrated that high doses of natural and SCs produced conditioned place aversion, ${ }^{72,73}$ whereas lower doses induced conditioned place preference. ${ }^{74,75}$ Furthermore, microinjections in the ventral tegmental area and nucleus 
accumbens produced conditioned place preference, an effect that was blocked by SR141716A (ie, rimonabant), a CB1 antagonist. Recently, Cha et al evaluated the psychological dependency potential of JWH-073, JWH-081, and JWH-210 using conditioned place preference, with significant dosedependent increases for the SCs administered groups. ${ }^{76}$ It must be noticed that SCs show a positive self-administration effect when the catheter was inserted directly into the ventral tegmental area, ${ }^{77}$ but failed to induce any effect by the venous route. ${ }^{57}$ Drug discrimination tests can establish a potential of abuse by comparing a substance to a well-known head file drug for its abuse potential. In such studies, CP47,497 generalizes for $\Delta^{9}$-THC in the rat, ${ }^{78} \mathrm{JWH}-018$ and JWH073 in the monkey, ${ }^{79}$ and JWH-200, JWH-203, JWH-250, AM-2201, and CP 47,497-C8-homologue in mice, with interesting differences in the duration of the effect. ${ }^{80} \mathrm{HU}-210$ generalizes for BAY 59-3074, a partial CB1/CB2 agonist in rat, and shows no effect when the CB1-antagonist is administrated. ${ }^{81}$

\section{Human use of SCs}

\section{Modalities of administration}

The herbal mixtures that are sprayed with SCs and are proposed as legal alternatives to marijuana are often smoked by users. ${ }^{2}$ Apart from herbal smoking blends, some consumers prefer homemade mixtures, using some "purified" powders of SCs sold on websites, solved in alcohol and spayed on herbals. With the recent development of electronic nicotine delivery system (e-cigarettes) as a new alternative for tobacco withdrawal, e-liquids containing SCs have recently appeared as a new trend and also as a more discreet way of consumption.

Ingestion of SCs is not often reported. One study detailed the cardiovascular effects on a man who had drunk SCs that was mixed with alcohol. ${ }^{82}$ A case series, including eleven users that ingested brownies laced with SCs, in the manner of the well-known "space-cake" consumption of cannabis, has been recently published. ${ }^{83}$ Other marginal routes of administration have been reported or have been discussed on user forum's such as nasal insufflation, either vaporized or not. MAM-2201 was recently quantified in the serum of a 20-yearold subject that snorted a powder sold as "Synthacaine". ${ }^{84}$ Administration by injection could theoretically produce the typical effects of SCs, but experimental data on mice suggest that differences can exist between inhalation and injection, especially in catalepsy and perhaps discriminative effects. ${ }^{85}$ Nevertheless, the injection route does not seem to be user's preference, partly due to the low water solubility of a number of SCs, leading to use some adjuvants. ${ }^{86}$
Dose used varied a lot between products, reported in experimental findings on SCs CB1 affinity. They fluctuate from several $\mathrm{mg}$ for the lead JWH-018, to several $\mu \mathrm{g}$ for the more potent HU-210. JWH-018 and JWH-073 were measured in a herbal incense product at concentrations of $17 \mathrm{mg} / \mathrm{g}$ and $22 \mathrm{mg} / \mathrm{g}$ respectively. ${ }^{87}$

\section{Recreational effects}

In humans, the recreational use of SCs generates psychoactive effects similar to cannabis, such as relaxation, calmness, euphoria, hilarity, lowering of inhibitions, disorientation, and an altered perception. Effects begin after only a few minutes from inhalation, and generally disappear after approximately 2-6 hours. ${ }^{2}$ SCs reportedly had both a shorter duration of action and a quicker time to peak onset of effect. ${ }^{88}$

\section{Acute and chronic, physical and psychological adverse/toxic effects}

After the use of SCs, it is essentially neurological and cardiovascular effects that essentially occur, with a prevalence of $61.9 \%$ and $43.5 \%$ respectively, reported from 464 cases at Texas poisoning centers over $2010 .{ }^{89}$ For the 305 adolescents, the medical outcome was known or suspected to be serious in $61 \%$ of these cases. In this young population, the most frequently reported adverse clinical effects were tachycardia (41.6\%), drowsiness/ lethargy (24.3\%), agitation/irritability (18.5\%), vomiting/ nausea (21.6\%), and hallucinations/delusions (10.8\%). Nausea, confusion, hypertension, chest pain, and dizziness/vertigo were observed in $<10 \%$ of the cases..$^{90}$ It is important to note that seizures were observed in $3.8 \%$ of the SCs intoxication cases, whereas they are not typically seen with marijuana use. ${ }^{91}$ Most of these effects lasted for $<8$ hours $(78.4 \%)$ and $92.9 \%$ of these cases did not have a life threatening clinical effect. ${ }^{92}$ Meanwhile, some evaluations on emergency medical treatments suggest that SCs potentially pose a greater risk to users' health than natural cannabinoids. ${ }^{93}$

In addition to acute SCs-induced psychosis disorders that include disorientation and hallucinations and can have life-threatening outcome, long-term effects also are to be feared. Although the long-term consequences of SC use are unclear, in patients with psychiatric disorders, new psychiatric phenomena could appear. Celofiga et al observed a marked worsening of mood and anxiety, without exacerbation of the pre-existing known psychotic symptoms. ${ }^{94}$ In 2015, Van Amsterdam et al showed that psychosis-inducing risk is higher with SCs than with natural cannabis. ${ }^{95}$ Hence, the psychosis outcomes associated with SCs provide additional data to the ongoing debate on cannabinoids and psychosis. ${ }^{96}$ 
In terms of physical damage there have been several recently reported cases; a severe pulmonary syndrome in an otherwise healthy young man which was related to habitual SCs smoking; ${ }^{97}$ a case of a cardiac arrest following K2 abuse; and a case of acute cerebral infarction in a 33 year old man, all close-temporally linked with the inhalation of XLR-11.98,99

Little is known on pregnancy/fertility impairment effects induced by SCs in humans due to limited studies, but regular cannabis consumption throughout pregnancy is statistically associated with decreases in birth weight. ${ }^{100}$ This data suggests that using SCs is a potential risk factor that could impact several stages of pregnancy. ${ }^{69}$

\section{Addictive potential}

In humans, tolerance to $\mathrm{SCs}$ has been reported in literature. ${ }^{101}$ The most common symptoms observed after an acute withdrawal are agitation, tachycardia, irritability, anxiety, and mood swings. In Auckland (New Zealand), patients withdrawing from SCs required intensive support, including medication and admission to an inpatient detoxification service. Between May 2013 and May 2014, SCs users represented the third largest group of patients admitted to this unit. Due to the stronger potency of some $\mathrm{SCs}$, withdrawal signs appeared more severe but did not seem to be improved by $\Delta^{9}$-THC. ${ }^{102,103}$ Furthermore, concurrently to a craving experienced by a 23 year old man, a user of "Spice gold", substantial but reversible short-term alterations of dopamine D2/3 receptor availability were shown in a PET Scan. ${ }^{104}$

\section{Pharmacokinetics}

\section{Concentrations in biological samples}

The JWH-018 and JWH-073 concentrations determined in postmortem whole blood samples varied from 0.1 to $199 \mu \mathrm{g} / \mathrm{L}$ and 0.1 to $68.3 \mu \mathrm{g} / \mathrm{L}$ respectively. ${ }^{105}$ Obtained in a patient who had smoked an herbal incense containing these two SCs, the concentrations fall from 4.8 and $4.2 \mu \mathrm{g} / \mathrm{L}$ (measured $=19$ minutes after dose administration) to 0.6 and $0.3 \mu \mathrm{g} / \mathrm{L}$ (measured $=107$ minutes after dose administration) for JWH-018 and JWH-073 respectively. ${ }^{87}$ The concentration of UR-144 in a blood sample collected on admission of a patient who was unconscious on arrival was $6.1 \mu \mathrm{g} / \mathrm{L}$. The parent compound was not found in urine but metabolites were identified. ${ }^{106} \mathrm{SCs}$ quantitated in twelve SCs serum users ranged from 0.21 to $2.94 \mu \mathrm{g} / \mathrm{L}$ for JWH-0250, and 0.35 to $73.05 \mu \mathrm{g} / \mathrm{L}$ for JWH-0122. ${ }^{16}$ In users driving under the influence of SCs, a very large difference was seen between the lowest $(0.07$, 0.08 , and $0.24 \mu \mathrm{g} / \mathrm{L}$, respectively) and the highest (4, 9.9, and
$24.5 \mu \mathrm{g} / \mathrm{L})$ blood concentrations of AM-2201, JWH-08, and APINACA. ${ }^{107}$

Wide variations were also observed for the quantitative results in 23 hair samples of SCs abuse suspects: 0.4 to $38.9 \mathrm{pg} / \mathrm{mg}$ for JWH-018, 0.1 to $0.8 \mathrm{pg} / \mathrm{mg}$ for JWH-073, 1.7 to $739.01 \mathrm{pg} / \mathrm{mg}$ for AM-2201, 0.1 to $402.0 \mathrm{pg} / \mathrm{mg}$ for JWH-122, and 0.2 to $276.0 \mathrm{pg} / \mathrm{mg}$ for MAM-2201. ${ }^{40}$ In two volunteers who smoked a joint prepared from different herbal incense products, the concentrations of measured SCs in neat oral fluid 5 hours later fluctuated between 0.1 and $1 \mu \mathrm{g} / \mathrm{L}$ for JWH-018 and JWH-210, but it was lower than $0.1 \mu \mathrm{g} / \mathrm{L}$ for JWH-200. ${ }^{33}$

\section{Metabolism}

Several recent publications characterized the metabolism pathways of SCs in vitro or identified degradant products in animal or human blood/urine samples. They all showed that SCs are extensively metabolized. SCs parent compounds are mainly hydroxylated, dealkylated, carboxylated, glucuronate. Furthermore, hydroxylation's take place on the aliphatic chain, the indole, the naphthalene, or the substituted aromatic rings that can be secondarily metabolized to carboxylic acids then conjugated to glucuronic acid. ${ }^{108,109}$ CYP3A4 has been recently demonstrated to be the major CYP enzyme responsible for the oxidative metabolism of AKB-48. ${ }^{110}$ Ashino et al suggested that SCs, especially naphthoylindole derivatives, are capable of inhibiting CYP1A enzymatic activity as do the major metabolites present in marijuana, cannabinol and cannabidiol. ${ }^{111}$

\section{Elimination rate}

The half-live of JWH-018 and JWH-073 calculated from the concentration data measured by Kacinko et al in a patient who had smoked an herbal incense containing these SCs, were 41 and 44 minutes respectively. ${ }^{87}$ Similar results were found after an incense JWH-018 smoking experiment performed on two healthy subjects, with the calculated half-live at 43 and 34 minutes. ${ }^{112}$ These data confirm the rapid decline of JWH-018 as early as the first puncture time at 5 minutes.

In another study an adult male volunteer orally ingested a $5 \mathrm{mg}$ dose of pure AM-2201, and the AM-2201 serum concentrations was reported to decreased from $1.4 \mu \mathrm{g} / \mathrm{L}$ at approximately 1 hour to $0.7 \mu \mathrm{g} / \mathrm{L}$ at 5 hours after ingestion. AM-2201 was still detectable in serum 25 hours after administration. The half-life of AM-2201 was estimated to be approximately 4 hours. ${ }^{33}$

After two volunteers smoked a mixture of SCs, JWH018, JWH-019, JWH-210, JWH-251, and JWH-307 were 
still detectable for approximately 26 hours in oral fluid; and JWH-251, JWH-210, and JWH-307 could be detected at 37 , 47 , and 55 hours, respectively. ${ }^{33}$

\section{Clinical management of acute and chronic adverse effects and addiction}

As for all other new psychoactive substances, there are limited reliable data to guide clinicians managing patients with toxicity due to SCs. As a consequence, management of SCs users with acute toxic effects is mainly extrapolated from experience with longer established cannabis effects.

\section{Diagnostic tests}

Routine laboratory tests in the work-up of a potentially SCs toxic patient include 1) a basic metabolic panel with blood glucose levels, serum electrolyte concentrations, liver and kidney function tests; 2) a complete blood cell count with coagulation studies; 3) cardiac markers; and 4) total creatinine kinase. The typically available urine toxicology screen will not detect SCs but could be useful to detect other possible substances ingested. If the patient presents signs and symptoms consistent with cannabis and concurrent with a natural cannabinoid screen negative, clinicians should suspect SCs use. More specific and sensitive chromatographic methods associated to MS would successful identify SCs. Electrocardiograms might be useful especially if the patient is profoundly tachycardic, and electroencephalogram might be indicated if a seizure is suspected or observed.

\section{Clinical exam}

In addition to the above mentioned laboratory tests, detailed physical and neurological exams are imperative.

The SCs which are high-affinity and high-efficacy agonists of the CB1 receptor mimic the "tetrad" of effects induced by cannabis in rodents. ${ }^{113}$ The common signs of SCs use are hallucinations, agitation, irritability, ${ }^{114}$ and psychotropic disturbances and paranoia have been also described. ${ }^{115}$ Myoclonia, seizure, nausea, vomiting, and hypokalemia may worsen the clinical status. ${ }^{115}$ Furthermore, cardiovascular effects such tachycardia/palpitation, hypertension, chest pain, and myocardial infarction, may occur. ${ }^{116}$ However, it is necessary to consider other well-known risk factors before formally linking SCs consumption to myocardial troubles. ${ }^{117}$

Due to the great variability of chemical classes that could be taken while using K2, Spice, or other mixes containing $\mathrm{SCs}$, unexpected toxic effects could also appear. For example, a case series of kidney injuries was collected in 2012 and was often linked, when identified, to XLR-11 and/or UR-144. ${ }^{118}$
Another unexpected clinical event, more potential than observed to our knowledge, due to the monoamine oxidase inhibitor activity of SCs demonstrated as real but weak in vitro, is serotoninergic syndrome that could be observed in patients, especially when high doses of SCs are taken by users. ${ }^{119}$ More recently, new physiopathological hypotheses could explain some of the undesired effects of SCs. For example, Irie et al observed that MAM-2201 is likely to suppress neurotransmitter release in $\mathrm{CB} 1$ receptor expressing neurons in mouse cerebellum purkinje cells, contributing to some of the symptoms of SCs intoxication including impairments in cerebellum-dependent motor coordination and motor learning. ${ }^{120}$ This kind of study is only the beginning step but it reinforces the need for vigilance to detect new toxicological syndromes in cases of suspicious SCs consumption. Faced with an unexplained clinical outcome, physicians have to be attentive in taking a proper history of the patient, with toxicological analysis (identification and quantification of the active substances in patient biological fluids as well as in taken drug), even if it is sometimes difficult to obtain. Also, physicians should always keep in mind that the combination of various SCs, which may be present in available products, and the taking of other illegal substances or alcohol could lead to unforeseen toxidromes.

\section{Treatment}

Considering that absorbed compounds are rarely identified and that clinical signs may be unspecific, no directive on the management of such patients has been formally promulgated by public health agencies. In fact, appropriate supportive care, targeting manifesting signs and symptoms and addressing any complications, is the primary treatment for the acutely intoxicated patient. ${ }^{121}$ Initial management should also include monitoring telemetry for arrhythmia, myocardial ischemia, serum electrolytes, and ensuring a secure airway. ${ }^{122}$ No specific antidote exists for SCs exposure and no curative treatment is approved by health authorities. The most common therapeutic intervention is hydration with intravenous fluids. ${ }^{92}$ Observation until the patient demonstrates clinical improvement is recommended and chemical dependency counseling or social service involvement should be considered before discharge. ${ }^{123}$

The course of treatment for SCs withdrawal is not well described in the literature. In Auckland, patients appearing at a detoxification service for support with SCs withdrawal and presenting withdrawal symptoms, are managed with benzodiazepine (diazepam) and antipsychotics (quetiapine). ${ }^{102}$ Based on a preclinical studies demonstrating a 
bidirectional modulatory relationship between opioid and cannabinoid systems, naloxone was tested to manage the SCs cravings in a 39 year old woman seeking detoxification for SC addiction, and could appear to be a potential option. ${ }^{124,125}$

\section{Legal status}

SCs have a different legal status around the world. Many states globally still schedule them "substance-by-substance" as new ones appear on the market, in an endless race against the resellers. ${ }^{10,126}$ On the other hand, some countries, UK being the first, have adopted a generic control status that has the great advantage of eluding the "cat and mouse game" problem. However, in the specific case of cannabimimetics that cover a large number of diversified chemical classes, it postpones the problem at one level but without solving it completely. Furthermore, generic control can pose the problem of unintended status for some substances that 1) are not CB1 agonist, and 2) show a medical interest, with an obstacle in the clinical development, as it has been seen in the UK. ${ }^{127}$

\section{Conclusion}

New psychoactive drugs that have appeared over the last decennia are typically dominated by cathinones and SCs. Commercialized as synthetic cannabis, SCs are sprayed onto various herbal smoking blends and traded under brand names like Spice or K2. More recently, SCs are also sold as pure substances ready to use with herbal mixtures or liquids for e-cigarettes. New compounds belonging to new chemical series are continuously emerging which generates a phenomenon that is very difficult to check. SCs pharmacologically act by binding to $\mathrm{CB} 1$ and/or $\mathrm{CB} 2$ receptors with $\mathrm{CB} 1$ agonists accountable for the recreational effects of SCs. The in vitro binding characteristics of SCs have been repeatedly determined, but the pharmacological properties are infrequently explored before human use. Along with unpleasant central nervous system effects, there are several physical adverse effects including kidney damage, pulmonary, gastrointestinal, and cardiovascular effects that can make consumption unsafe. Even if most of the SCs mimic marijuana effects, some SCs bind much more strongly to CB1 receptors than natural cannabinoids, which can lead to more potent, unpredictable, or dangerous effects. Because of the multitude of compounds, a complete toxicological profile of SCs is far from being drawn and understood. In addition, these SCs are often taken in conjunction with other recreational drugs or alcohol, which makes observed effects difficult to attribute to a specific product. Despite their status being illegal in some countries, SCs continue to be prevalent drugs of abuse with collateral damage such as increased road traffic risks. Accordingly, clinicians should be aware of this developing trend as an explanation for patients presenting unexpected toxidromes. Standard routine toxicology screens, particularly targeted for cannabis detection, may not detect the presence of these compounds. More specific methods may be required for identification and quantification in biological samples, with the ever-increasing diversity of new products making the updates unrealizable for the most part of toxicological laboratories. Meanwhile, clinicians should remain attentive for the risk of increasing morbidity and mortality associated to these products, continue to collect and publish new trends about these highs, and help promote awareness in their communities.

\section{Disclosure}

The authors report no conflicts of interest in this work.

\section{References}

1. Domino EF, Hardman HF, Seevers MH. Central nervous system actions of some synthetic tetrahydrocannabinol derivatives. Pharmacol Rev. 1971;23(4):317-336.

2. Auwärter V, Dresen S, Weinmann W, Müller M, Pütz M, Ferreirós N. 'Spice' and other herbal blends: harmless incense or cannabinoid designer drugs? J Mass Spectrum. 2009;44(5):832-837.

3. Castaneto MS, Gorelick DA, Desrosiers NA, Hartman RL, Pirard S, Huestis MA. Synthetic cannabinoids: epidemiology, pharmacodynamics, and clinical implications. Drug Alcohol Depend. 2014;144:12-41.

4. Mackie K. Cannabinoid receptors: where they are and what they do. J Neuroendocrinol. 2008;20(Suppl 1):10-14.

5. Pertwee RG. Cannabinoid pharmacology: the first 66 years. Br J Pharmacol. 2006;147(Supp11):S163-S171.

6. Johnson MR, Melvin LS. The discovery of non-classical cannabinoid analgesics. In: Mechoulam R, editor. Cannabinoids as Therapeutic Agents. CRC Press, Boca Raton: Florida. 1986:121-145.

7. Huffman JW, Dong D. Synthesis and pharmacology of cannabimimetic indoles. Bioorg Med Chem Lett. 1994;4(4):563-566.

8. Lindigkeit R, Boehme A, Eiserloh I, et al. Spice : a never ending story? Forensic Sci Int. 2009;191(1-3):58-63.

9. Uchiyama N, Kikura-Hanajiri R, Ogata J, Goda Y. Chemical analysis of synthetic cannabinoids as designer drugs in herbal products. Forensic Sci Int. 2010;198(1-3):31-38.

10. European Monitoring Centre for Drugs and Drug Addiction [website on the Internet]. Drug profiles. Availableat: http://www.emcdda.europa. eu/publications/drug-profiles. Accessed April 8, 2015.

11. Wohlfarth A, Scheidweiler KB, Castaneto M, et al. Urinary prevalence, metabolite detection rates, temporal patterns and evaluation of suitable LC-MS/MS targets to document synthetic cannabinoid intake in US military urine specimens. Clin Chem Lab Med. 2015;53(3):423-434.

12. Palamar JJ, Acosta P. Synthetic cannabinoid use in a nationally representative sample of US high school seniors. Drug Alcohol Depend. 2015;149:194-202.

13. Kelly BC, Wells BE, Pawson M, Leclair A, Parsons JT, Golub SA. Novel psychoactive drug use among younger adults involved in US nightlife scenes. Drug Alcohol Rev. 2013;32(6):588-593.

14. Hu X, Primack BA, Barnett TE, Cook RL. College students and use of K2: an emerging drug of abuse in young persons. Subst Abuse Treat Prev Policy. 2011;6:16.

15. Egan KL, Suerken CK, Reboussin BA, et al. K2 and Spice use among a cohort of college students in southeast region of the USA. Am J Drug Alcohol Abuse. 2015;41(4):317-322. 
16. Jaenicke NJ, Pogoda W, Paulke A, Wunder C, Toennes SW. Retrospective analysis of synthetic cannabinoids in serum samples - epidemiology and consumption patterns. Forensic Sci Int. 2014;242:81-87.

17. Beck F, Richard JB, Guignard R, Le Nézet O, Spilka S. Illicit drugs used in France during 2014. Note 2015-01. OFDT- INPES. http://www.ofdt.fr/ BDD/publications/docs/eisxfbv4.pdf. Accessed April 15, 2015 French.

18. Louis A, Peterson BL, Couper FJ. XLR-11 and UR-144 in Washington state and state of Alaska driving cases. J Anal Toxicol. 2014;38(8): 563-568.

19. Lemos NP. Driving under the influence of synthetic cannabinoid receptor agonist XLR-11. J Forensic Sci. 2014;59(6):1679-1683.

20. Tuv SS, Krabseth H, Karinen R, Olsen KM, Øiestad EL, Vindenes V. Prevalence of synthetic cannabinoids in blood samples from Norwegian drivers suspected of impaired driving during a seven weeks period. Accid Anal Prev. 2014;62:26-31.

21. Adamowicz P, Lechowicz W. The Influence of Synthetic Cannabinoid UR-144 on Human Psychomotor Performance - A Case Report Demonstrating Road Traffic Risks. Traffic Inj Prev. Epub 2015 Mar 20.

22. More SV, Choi DK. Promising cannabinoid-based therapies for Parkinson's disease: motor symptoms to neuroprotection. Mol Neurodegener. 2015;10:17.

23. Picone RP, Kendall DA Mol Endocrinol. Minireview: From the Bench, toward the clinic: therapeutic opportunities for cannabinoid receptor modulation. Mol Endocrinol. 2015;29(6):801-813.

24. Arnston A, Ofsa B, Lancaster D, Simon JR, Mc Mullin M, Logan B. Validation of a novel immunoassay for the detection of synthetic cannabinoids and metabolites in urine specimens. J Anal Toxicol. 2013;37(5):284-290

25. Mohr A, Ofsa B, Keil A, Simon J, McMullin M, Logan B. Enzyme-Linked Immunosorbent Assay (ELISA) for the detection of use of the synthetic cannabinoid agonists UR-144 and XLR-11 in human urine. J Anal Toxicol. 2014;38(7):427-431.

26. Barnes AJ, Young S, Spinelli E, Martin TM, Klette KL, Huestis MA. Evaluation of a homogenous enzyme immunoassay for the detection of synthetic cannabinoids in urine. Forensic Sci Int. 2014;241:27-34.

27. Simolka K, Lindigkeit R, Schiebel HM, Papke U, Ernst L, Beuerle T. Analysis of synthetic cannabinoids in "spice-like" herbal highs: snapshot of the German market in summer 2011. Anal Bioanal Chem 2012;404(1):157-171.

28. Cox AO, Daw RC, Mason MD, et al. Use of SPME-HS-GC-MS for the analysis of herbal products containing synthetic cannabinoids. J Anal Toxicol. 2012;36(5):293-302.

29. Moosmann B, Kneisel S, Girreser U, Brecht V, Westphal F, Auwärter V. Separation and structural characterization of the synthetic cannabinoids JWH412 and 1-[(5-fluoropentyl)-1H-indol-3yl]-(4-methylnaphthalen1-yl)methanone using GC-MS, NMR analysis and a flash chromatography system. Forensic Sci Int. 2012;220(1-3):e17-e22.

30. Choi H, Heo S, Choe S, et al. Simultaneous analysis of synthetic cannabinoids in the materials seized during drug trafficking using GC-MS Anal Bioanal Chem. 2013;405(12):3937-3944.

31. Langer N, Lindigkeit R, Schiebel HM, Ernst L, Beuerle T. Identification and quantification of synthetic cannabinoids in 'spice-like' herbal mixtures: a snapshot of the German situation in the autumn of 2012. Drug Test Anal. 2014;6(1-2):59-71.

32. Kneisel S, Bisel P, Brecht V, Broecker S, Müller M, Auwärter V. Identification of the cannabimimetic AM-1220 and its azepane isomer ( $N$-methylazepam-3yl)-3-(1-naphthoyl)indole in research chemical and several herbal mixtures. Forensic Toxicol. 2012:30;126-134.

33. Kneisel S, Speck M, Moosmann B, Corneillie TM, Butlin NG, AuwärterV. LC/ESI-MS/MS method for quantification of 28 synthetic cannabinoids in neat oral fluid and its application to preliminary studies on their detection windows. Anal Bioanal Chem. 2013;405(14):4691-4706.

34. Dziadosz M, Weller JP, Klintschar M, Teske J. Scheduled multiple reaction monitoring algorithm as a way to analyze new designer drugs combined with synthetic cannabinoids in human serum with liquid chromatography-tandem mass spectrometry. J Chromatogr B Analyt Technol Biomed Life Sci. 2013;929:84-89.
35. Derungs A, Schwaninger AE, Mansella G, Bingisser R, Kraemer T, Liechti ME. Symptoms, toxicities, and analytical results for a patient after smoking herbs containing the novel synthetic cannabinoid MAM2201. Forensic Toxicology. 2013;31(1):164-171.

36. Salomone A, Luciano C, Di Corcia D, Gerace E, Vincenti M. Hair analysis as a tool to evaluate the prevalence of synthetic cannabinoids in different populations of drug consumers. Drug Test Anal. 2014;6(1-2): 126-134.

37. Scheidweiler KB, Huestis MA. Simultaneous quantification of 20 synthetic cannabinoids and 21 metabolites, and semi-quantification of 12 alkyl hydroxy metabolites in human urine by liquid chromatographytandem mass spectrometry. J Chromatogr A. 2014;1327:105-117.

38. Mazzarino M, de la Torre X, Botrè F. A liquid chromatography-mass spectrometry method based on class characteristic fragmentation pathways to detect the class of indole-derivative synthetic cannabinoids in biological samples. Anal Chim Acta. 2014;837:70-82.

39. Simões SS, Silva I, Ajenjo AC, Dias MJ. Validation and application of an UPLC-MS/MS method for the quantification of synthetic cannabinoids in urine samples and analysis of seized materials from the Portuguese market. Forensic Sci Int. 2014;243:117-125.

40. Kim J, Park Y, Park M, et al. Simultaneous determination of five naphthoylindole-based synthetic cannabinoids and metabolites and their deposition in human and rat hair. J Pharm Biomed Anal. 2015;102: 162-175.

41. Jang M, Shin I, Kim J, Yang W. Simultaneous quantification of 37 synthetic cannabinoid metabolites in human urine by liquid chromatography-tandem mass spectrometry. Forensic Toxicol. 2015. DOI 10.1007/ s11419-015-0265-x

42. Gottardo R, Chiarini A, Dal Prà I, et al. Direct screening of herbal blends for new synthetic cannabinoids by MALDI-TOF MS. J Mass Spectrom. 2012;47(1):141-146.

43. Sundström M, Pelander A, Angerer V, Hutter M, Kneisel S, Ojanperä I. A highsensitivity ultra-high performance liquid chromatography/highresolution timeof-flight mass spectrometry (UHPLC-HR-TOFMS) method for screening synthetic cannabinoids and other drugs of abuse in urine. Anal Bioanal Chem. 2013;405(26):8463-8474.

44. Kronstrand R, Brinkhagen L, Birath-Karlsson C, Roman M, Josefsson M. LCQTOF-MS as a superior strategy to immunoassay for the comprehensive analysis of synthetic cannabinoids in urine. Anal Bioanal Chem. 2014;406(15):3599-3609.

45. Gottardo R, Sorio D, Musile G, et al. Screening for synthetic cannabinoids in hair by using LC-QTOF MS: a new and powerful approach to study the penetration of these new psychoactive substances in the population. Med Sci Law. 2014;54(1):22-27.

46. Musah RA, Domin MA, Walling MA, Shepard JR. Rapid identification of synthetic cannabinoids in herbal samples via direct analysis in real time mass spectrometry. Rapid Commun Mass Spectrom. 2012;26(9): 1109-1114.

47. Dowling G, Regan L. A method for CP 47, 497 a synthetic nontraditional cannabinoid in human urine using liquid chromatography tandem mass spectrometry. J Chromatogr B Analyt Technol Biomed Life Sci. 2011;879(3-4):253-259.

48. Callén L, Moreno E, Barroso-Chinea P, et al. Cannabinoid receptors $\mathrm{CB}_{1}$ and $\mathrm{CB}_{2}$ form functional heteromers in brain. J Biol Chem. 2012; 287(25):20851-20865.

49. Blankman JL, Cravatt BF. Chemical probes of endocannabinoid metabolism. Pharmacol Rev. 2013;65(2):849-871.

50. Abood ME. Molecular biology of cannabinoid receptors: mutational analyses of the $\mathrm{CB}$ receptors. In Reggio $\mathrm{PH}$. The cannabinoids receptors. Humana Press. Greensboro, NC; 2009:203-234.

51. Pertwee RG. Pharmacological actions of cannabinoids. Handbook of Experimental Pharmacologyed. Heidelburg-Verlag. 2005;(168):1-51.

52. Huffman JW, Zengin G, Wu MJ, et al. Structure-activity relationships for 1-alkyl-3-(1-naphthoyl)indoles at the cannabinoid $\mathrm{CB}(1)$ and $\mathrm{CB}(2)$ receptors: steric and electronic effects of naphthoyl substituents. New highly selective $\mathrm{CB}(2)$ receptor agonists. Bioorg Med Chem. 2005;13(1): 89-112. 
53. Aung MM, Griffin G, Huffman JW, et al. Influence of the N-1 alkyl chain length of cannabimimetic indoles upon $\mathrm{CB}(1)$ and $\mathrm{CB}(2)$ receptor binding. Drug Alcohol Depend. 2000;60(2):133-140.

54. Makriyannis A, Deng H. WO patent 200128557. Cannabimimetic indole derivatives. Granted 2001-06-07.

55. Huffman JW, Szklennik PV, Almond A, et al. 1-Pentyl-3-phenylacetylindoles, a new class of cannabimimetic indoles. Bioorg Med Chem Lett. 2005;15(18):4110-4113.

56. Frost JM, Dart MJ, Tietje KR, et al. Indol-3ylcycloalkyl ketones: effects of $\mathrm{N} 1$ substituted indole side chain variations on $\mathrm{CB}(2)$ cannabinoid receptor activity. J Med Chem. 2010;53(1):295-315.

57. McGaraughty, S, Chu KL, Dart MJ, Yao BB, Meyer MD. A CB(2) receptor agonist, A-836339, modulates wide dynamic range neuronal activity in neuropathic rats: contributions of spinal and peripheral $\mathrm{CB}(2)$ receptors. Neuroscience 2009;158(4):1652-1661.

58. Deng H, Gifford AN, Zvonok AM, et al. Potent cannabinergic indole analogues as radioiodinatable brain imaging agents for the CB1 cannabinoid receptor. $J$ Med Chem. 2005;48(20):6386-6392.

59. Smith VJ. Synthesis and pharmacology of N-alkyl-3-(halonaphtoyl) indoles. [PhD dissertation]. Clemson University, South Carolina, USA; 2008.

60. Abadji V, Lin S, Taha G, et al. (R)-methanandamide: a chiral novel anandamide possessing higher potency and metabolic stability. $J$ Med Chem. 1994;37(12):1889-1893.

61. Huffmann JW, Smith VJ, Chen J, Wiley JL, Martin BR. Structure-activity relationships at the CB1 and CB2 receptors for 1-alkyl-3(1-naphtoyl-4 and 8-halogen substituted) indoles. 19th Annual Symposium on the International Cannabinoid Research Society. July 7-11, 2009; St Charles, Illinois, USA. Page 2.

62. Wrobleski ST, Chen P, Hynes J Jr, et al. Rational design and synthesis of an orally active indolopyridone as a novel conformationally constrained cannabinoid ligand possessing anti-inflammatory properties. $J$ Med Chem. 2003;46(11):2110-2116.

63. Huffmann. Cannabimimetic indoles, pyrroles, and Indenes: structureactivity relationships and receptor interactions. In: Reggio PH. The cannabinoids receptors. Humana Press. 2009:49-94.

64. Makriyannis A, Lai XZ, Lu, D. Novel biphenyl and biphenyl-like cannabinoids. Patent US20040087590 A1. May 6, 2004.

65. Console-Bram L, Marcu J, Abood ME. Cannabinoid receptors: nomenclature and pharmacological principles. Prog Neuropsychopharmacol Biol Psychiatry. 2012;38(1):4-15.

66. Pertwee RG. The diverse $\mathrm{CB}_{1}$ and $\mathrm{CB}_{2}$ receptor pharmacology of three plant cannabinoids: $\Delta^{9}$-tetrahydrocannabinol, cannabidiol and $\Delta^{9}$-tetrahydrocannabivarin. Br J Pharmacol. 2008;153(2):199-215.

67. Howlett AC. The cannabinoid receptors. Prostaglandins Other Lipid Mediat. 2002;68-69:619-631.

68. Pacher P, Batkai S, Kunos G. The endocannabinoid system as an emerging target of pharmacotherapy. Pharmacol Rev. 2006;58(3):389-462.

69. Sun X, Dey SK. Synthetic cannabinoids and potential reproductive consequence. Life Sci. 2014;97(1):72-77.

70. Ng Cheong Ton JM, Gerhardt GA, Friedemann M, et al. The effects of delta 9-tetrahydrocannabinol on potassium-evoked release of dopamine in the rat caudate nucleus: an in vivo electrochemical and in vivo microdialysis study. Brain Res. 1988;451(1-2):59-68.

71. French ED, Dillon K, Wu X. Cannabinoids excite dopamine neurons in the ventral tegmentum and substantia nigra. Neuroreport. 1997;8(3):649-652.

72. Parker LA, Gillies T. THC-induced place and taste aversions in Lewis and Sprague-Dawley rats. Behav Neurosci. 1995:109(1):71-78

73. McGregor IS, Issakidis CN, Prior G. Aversive effects of the synthetic cannabinoid CP 55,940 in rats. Pharmacol Biochem Behav. 1996;53(3): 657-664.

74. Lepore M, Vorel SR, Lowinson J, Gardner EL. Conditioned place preference induced by delta 9-tetrahydrocannabinol: comparison with cocaine, morphine, and food reward. Life Sci. 1995;56(23-24): 2073-2080
75. Braida D1, Pozzi M, Cavallini R, Sala M. Conditioned place preference induced by the cannabinoid agonist CP 55,940: interaction with the opioid system. Neuroscience. 2001;104(4):923-926.

76. Cha HJ, Lee KW, Song MJ, et al. Dependence Potential of the Synthetic Cannabinoids JWH-073, JWH-081, and JWH-210: In Vivo and In Vitro Approaches. Biomol Ther (Seoul).2014;22(4):363-369.

77. Zangen A, Solinas M, Ikemoto S, Goldberg SR, Wise RA. Two brain sites for cannabinoid reward. J Neurosci. 2006;26(18): 4901-4907.

78. Weissman A, Milne GM, Melvin LS Jr. Cannabimimetic activity from CP47,497, a derivative of 3-phenylcyclohexanol. J Pharmacol Exp Ther. 1982;223(2):516-523.

79. Ginsburg BC, Schulze DR, Hruba L, McMahon LR. JWH-018 and JWH-073: $\Delta^{9}$-tetrahydrocannabinol-like discriminative stimulus effects in monkeys. J Pharmacol Exp Ther. 2012;340(1):37-45.

80. Gatch MB, Forster MJ. $\Delta^{9}$-Tetrahydrocannabinol-like discriminative stimulus effects of compounds commonly found in $\mathrm{K} 2 / \mathrm{Spice}$. Behav Pharmacol. 2014;25(8):750-757.

81. De Vry J, Jentzsch KR. Discriminative stimulus effects of the structurally novel cannabinoid CB1/CB2 receptor partial agonist BAY 59-3074 in the rat. Eur J Pharmacol. 2004;505(1-3):127-133.

82. Lapoint J, James LP, Moran CL, Nelson LS, Hoffman RS, Moran JH. Severe toxicity following synthetic cannabinoid ingestion. Clin Toxicol (Phila). 2011;49(8):760-764.

83. Obafemi AI, Kleinschmidt K, Goto C, Fout D. Cluster of acute toxicity from ingestion of synthetic cannabinoid-laced brownies. J Med Toxicol. Epub 2015.

84. Lonati D, Buscaglia E, Papa P, et al. MAM-2201 (analytically confirmed) intoxication after "Synthacaine" consumption. Ann Emerg Med. 2014;64(6):629-632.

85. Marshell R, Kearney-Ramos T, Brents LK, et al. In vivo effects of synthetic cannabinoids JWH-018 and JWH073 and phytocannabinoid $\Delta^{9}$-THC in mice: inhalation versus intraperitoneal injection. Pharmacol Biochem Behav. 2014;124:40-47.

86. BLULIGHT [forum on the Internet]. Other drugs Forum. Available from: http://www.bluelight.org/vb/threads/539892-Injection-ofsyntheticcannabinoids-like-a-JWH. Accessed May 13, 2015.

87. Kacinko SL, Xu A, Homan JW, McMullin MM, Warrington DM, Logan BK. Development and validation of a liquid chromatographytandem mass spectrometry method for the identification and quantification of JWH-018, JWH-073, JWH-019, and JWH-250 in human whole blood. J Anal Toxicol. 2011;35(7):386-393.

88. Winstock AR, Barratt MJ. Synthetic Cannabis: A comparison of patterns of use and effect profile with natural Cannabis in a large global sample. Drug Alcohol Depend. 2013;131(1-2):106-111.

89. Forrester MB, Kleinschmidt K, Schwarz E, Young A. Synthetic cannabinoid exposures reported to Texas poison centers. J Addict Dis. 2011;30(4):351-358.

90. Forrester MB. Adolescent synthetic cannabinoid exposures reported to Texas poison centers. Pediatr Emerg care. 2012;28(10):985-989.

91. de Havenon A, Chin B, Thomas KC, Afra P. The secret "spice": an undetectable toxic cause of seizure. Neurohospitalist. 2011;1(4):182-186.

92. Hoyte CO, Jacob J, Monte AA, Al-Jumaan M, Bronstein AC, Heard KJ. A characterization of synthetic cannabinoid exposures reported to the National Poison Data System in 2010. Ann Emerg Med. 2012;60(4): 435-438.

93. Winstock A, Lynskey M, Borschmann R, Waldron J. Risk of emergency medical treatment following consumption of Cannabis or synthetic cannabinoids in a large global sample. J Psychopharmacol. 2015;29(6):698-703.

94. Celofiga A, Koprivsek J, Klavz J. Use of synthetic cannabinoids in patients with psychotic disorders: case series. J Dual Diagn. 2014;10(3): 168-173.

95. Van Amsterdam J, Brunt T, van den Brink W. The adverse health effects of synthetic cannabinoids with emphasis on psychosis-like. J Psychopharmacol. 2015;29(3):254-263. 
96. Spaderna M, Addy PH, D'Souza DC. Spicing things up: synthetic cannabinoids. Psychopharmacol (Berl). 2013;228(4):525-540.

97. Alhadi S, Tiwari A, Vohra R, Gerona R, Acharya J, Bilello K. High times, low sats: diffuse pulmonary infiltrates associated with chronic synthetic cannabinoid use. J Med Toxicol. 2013;9(2):199-206.

98. Ibrahim S, Al-Saffar F, Wannenburg T. A unique case of cardiac arrest following K2 abuse. Case report Cardiologia. 2014:120607.

99. Takematsu M, Hoffman RS, Nelson LS, Schechter JM, Moran JH, Wiener SW. A case of acute cerebral ischemia following inhalation of a synthetic cannabinoid. Clin Toxicol (Phila). 2014;52(9):973-975.

100. Fergusson DM, Horwood LJ, Northstone K. Maternal use of cannabis and pregnancy outcome. BJOG. 2002;109(1):21-27.

101. Every-Palmer S. Synthetic cannabinoid JWH-018 and psychosis: an explorative study. Drug Alcohol Depend. 2011;117(2-3):152-157.

102. Macfarlane V, Christie G. Synthetic cannabinoid withdrawal: a new demand on detoxification services. Drug Alcohol Rev. 2015;34(2):147-153.

103. Nacca N, Vatti D, Sullivan R, Sud P, Su M, Marraffa J. The synthetic cannabinoid withdrawal syndrome. J Addict Med. 2013;7(4): 296-298.

104. Rominger A, Cumming P, Xiong G, et al. Effects of acute detoxification of the herbal blend 'Spice Gold' on dopamine D2/3 receptor availability: a [18F]fallypride PET study. Eur Neuropsychopharmacol. 2013;23(11):1606-1610.

105. Shanks KG, Daahn T, Terrel AR. Detection of JWH-018 and JWH073 by UPLC-MS-MS in postmortem whole blood casework. J Anal Toxicol. 2012;36(3):145-152.

106. Adamowicz P, Zuba D, Sekula K. Analysis of UR-144 and its pyrolysis product in blood and their metabolites in urine. Forensic Sci Int 2013;233:320-327.

107. Karinen R, Tuv SS, Øiestad EL, Vindenes V. Concentrations of APINACA, 5F-APINACA, UR-144 and its degradant product in blood samples from six impaired drivers compared to previous reported concentrations of other synthetic cannabinoids. Forensic Sci Int 2015;246:98-103.

108. Gambaro V, Arnoldi S, Bellucci S, et al. Characterization of in vitro metabolites of JWH-018, JWH-073 and their 4-methyl derivatives, markers of the abuse of these synthetic cannabinoids. $J$ Chromatogr B Analyt Technol Biomed Life Sci. 2014;957:68-76.

109. Strano-Rossi S, Anzillotti L, Dragoni S, et al. Metabolism of JWH-015, JWH-098, JWH-251, and JWH-307 in silico and in vitro: a pilot study for the detection of unknown synthetic cannabinoids metabolites. Anal Bioanal Chem. 2014;406(15):3621-3636.

110. Holm NB, Nielsen LM, Linnet K. CYP3A4 mediates oxidative metabolism of the synthetic cannabinoid AKB-48. AAPS J. Epub 2015.

111. Ashino T, Hakukawa K, Itoh Y, Numazawa S. Inhibitory effect of synthetic cannabinoids on CYP1A activity in mouse liver microsomes. J Toxicol Sci. 2014;39(6):815-820.

112. Teske J, Weller JP, Fieguth A, Rothämel T, Schulz Y, Tröger HD. Sensitive and rapid quantification of the cannabinoid receptor agonist naphthalen-1-yl-(1-pentylindol-3yl)methanone (JWH-018) in human serum by liquid chromatography-tandem mass spectrometry. J Chromatogr B Analyt Technol Biomed Life Sci. 2010;878(27): 2659-2663.
113. Wiebelhaus JM, Poklis JL, Poklis A, Vann RE, Lichtman AH, Wise LE. Inhalation exposure to smoke from synthetic "marijuana" produces potent cannabimimetic effects in mice. Drug Alcohol Depend 2012;126(3):316-323.

114. Brewer TL, Collins M. A review of clinical manifestation in adolescent and young adults after use of synthetic cannabinoids. J Spec Pediatr Nurs 2014;19(2):119-126.

115. Hermanns-Clausen M, Kneisel S, Szabo B, et al. Acute toxicity due to the confirmed consumption of synthetic cannabinoids: clinical and laboratory findings. Addiction. 2013;108(3):534-544.

116. Mir A, Obafemi A, Young A, Kane C. Myocardial infarction associated with use of the synthetic cannabinoid K2. Pediatrics. 2011;128(6):e1622-e1627.

117. Alexandre J, Debruyne D, Coquerel A, Le Boisselier R. Comment on A Unique Case of Cardiac Arrest following K2 Abuse. Case Rep Cardiol. 2015;2015:739149.

118. Centers for Disease Control and Prevention (CDC). Acute kidney injury associated with cannabinoid use in multiple states, 2012. Morb Mortal Wkly Rep. 2013;62(6):93-98.

119. Fisar Z. Inhibition of monoamine oxidase activity by cannabinoids Naunyn Schmiedebergs Arch Pharmacol. 2010;381(6):563-572.

120. Irie T, Kikura-Hanajiri R, Usami M, Uchiyama N, Goda Y, Sekino Y. MAM-2201, a synthetic cannabinoid drug of abuse, suppresses the synaptic input to cerebellar Purkinje cells via activation of presynaptic CB1 receptors. Neuropharmacology. 2015;95:479-491.

121. Weaver MF, Hopper JA, Gunderson EW. Designer drugs 2015: assessment and management. Addict Sci Clin Pract. 2015;10:8.

122. Health TS, Burroughs Z, Thompson AJ, Tecklenburg FW. Acute intoxication caused by a synthetic cannabinoid in two adolescents. J Pediatr Pharmacol Ther. 2012;17(2):177-181.

123. Harris CR, Brown A. Synthetic cannabinoid intoxication. J Emerg Med. 2013;44(2):360-366.

124. Rodgman CJ, Verrico CD, Worthy RB, Lewis EE. Inpatient detoxification from a synthetic cannabinoid and control of postdetoxification cravings with naltrexone. Prim Care Companion CNS Disord. 2014;16(4).

125. Robledo P, Berrendero F, Ozaita A, Maldonado R. Advances in the field of cannabinoid-opioid cross-talk. Addict Biol. 2008;13(2):213-224.

126. Drug Enforcement Administration, Department of Justice. Schedules of controlled substances: temporary placement of three synthetic cannabinoids into schedule I. Final order. Fed Regist. 2015;80(20): 5042-5047.

127. King LA. Legal controls on cannabimimetics: an international dilemma? Drug Test Anal. 2014;6(1-2):80-87.

128. Shevyrin V, Melkozerov V, Nevero A, Eltsov O, Baranovsky A, Shafran Y. Synthetic cannabinoids as designer drugs: new representatives of indol-3carboxylates series and indazole-3-carboxylates as novel group of cannabinoids. Identification and analytical data Forensic Sci Int. 2014;244:263-275.
Substance Abuse and Rehabilitation

\section{Publish your work in this journal}

Substance Abuse and Rehabilitation is an international, peer-reviewed, open access journal publishing original research, case reports, editorials, reviews and commentaries on all areas of addiction and substance abuse and options for treatment and rehabilitation. The manuscript management system is completely online and includes a very quick and fair

\section{Dovepress}

peer-review system. Visit http://www.dovepress.com/testimonials.php to read real quotes from published authors. 